\title{
TRIMAGE: a dedicated trimodality (PET/MR/EEG) imaging tool for schizophrenia
}

Alberto Del Guerra $\left(1,{ }^{*}\right)$, Salleh Ahmad (2), Mihai Avram $(3,4,5)$, Nicola Belcari (1), Arne Berneking (6), Laura Biagi (7), Maria Giuseppina Bisogni (1), Felix Brandl (4,5), Jorge Cabello (3), Niccolò Camarlinghi (1), Piergiorgio Cerello (8), Chang-Hoon Choi (6), Silvia Coli (8), Sabrina Colpo (9), Julien Fleury (2), Vito Gagliardi (1), Giuseppe Giraudo (8), Karsten Heekeren (10), Wolfram Kawohl (10,11), Theodora Kostou (12), Jean-Luc Lefaucheur (13), Christoph Lerche (6), George Loudos (12), Matteo Morrocchi (1), Julien Muller (14), Mona Mustafa (3), Irene Neuner $(6,14)$, Panagiotis Papadimitroulas (12), Francesco Pennazio (8), Ravichandran Rajkumar (6,15), Cláudia Régio Brambilla (6), Julien Rivoire (14), Elena Rota Kops (6), Jürgen Scheins (6), Rémy Schimpf (14), N. Jon Shah (6), Christian Sorg (4,5,17), Giancarlo Sportelli (1), Michela Tosetti (7), Riccardo Trinchero (8), Christine Wyss (10), Sibylle Ziegler (3,16); TRIMAGE Consortium (18)

(1) Dipartimento di Fisica "E. Fermi", Università di Pisa, and INFN, Sezione di Pisa, Pisa, Italy

(2) Weeroc s.a.s., Palaiseau, France

(3) Nuklearmedinische Klinik und Poliklinik, Klinikum rechts der Isar, Technische Universität München, Munich, Germany

(4) Department of Neuroradiology, Klinikum rechts der Isar, Technische Universität München, Munich, Germany

(5) Neuroimaging Center (TUM-NIC), Klinikum rechts der Isar, Technische Universität München, Munich, Germany

(6) Forschungszentrum Jülich GmbH, Institute of Neuroscience and Medicine, INM4, Jülich, Germany

(7) IRCSS, Stella Maris, Calambrone, Pisa, Italy

(8) Istituto Nazionale di Fisica Nucleare, Sezione di Torino, Torino, Italy

(9) Advansid s.r.l., Povo, Trento, Italy

(10) Department of Psychiatry, Psychotherapy and Psychosomatics, University Hospital of Psychiatry Zurich, University of Zurich, Switzerland

(11) Department of Psychiatry and Psychotherapy, Psychiatric Services of Aargovia, Switzerland

(12) Technological Educational Institute of Athens, Greece

(13) Inviscan s.a.s., France

(14) RS2D s.a.s., Mundolsheim, France

(15) Department of Psychiatry, Psychotherapy and Psychosomatics, Faculty of Medicine, RWTH Aachen University, JARA Brain, Aachen, Germany

(16) Department of Nuclear Medicine, University Hospital, LMU, Munich, Germany

(17) Department of Psychiatry, Klinikum rechts der Isar, Technische Universität München, Munich, Germany

(18) www.trimage.eu

(*) Corresponding Author: Alberto Del Guerra, Department of Physics, Pisa University. TRIMAGE coordinator, e-mail: alberto.del.guerra@unipi.it

Keywords: Schizophrenia, Biomarkers, Trimodal imaging, PET, MR, EEG 


\begin{abstract}
Simultaneous PET/MR/EEG (Positron Emission Tomography - Magnetic Resonance Electroencephalography), a new tool for the investigation of neuronal networks in the human brain, is presented here within the framework of the European Union Project TRIMAGE. The trimodal, cost-effective PET/MR/EEG imaging tool makes use of cutting edge technology both in PET and in MR fields. A novel type of magnet (1.5T, non-cryogenic) has been built together with a PET scanner that makes use of the most advanced photodetectors (i.e., SiPM matrices), scintillators matrices (LYSO) and digital electronics. The combined PET/MR/EEG system is dedicated to brain imaging and has an inner diameter of $260 \mathrm{~mm}$ and an axial Field-of-View of $160 \mathrm{~mm}$.

It enables the acquisition and assessment of molecular metabolic information with high spatial and temporal resolution in a given brain simultaneously. The dopaminergic system and the glutamatergic system in schizophrenic patients are investigated via PET, the same physiological / pathophysiological conditions with regard to functional connectivity, via fMRI, and its electrophysiological signature via EEG. In addition to basic neuroscience questions addressing neurovascular-metabolic coupling, this new methodology lays the foundation for individual physiological and pathological fingerprints for a wide research field addressing healthy aging, gender effects, plasticity and different psychiatric and neurological diseases.

The preliminary performances of two components of the imaging tool (PET and MR) are discussed. Initial results of the search of possible candidates for suitable schizophrenia biomarkers are also presented as obtained with PET/MR systems available to the collaboration.
\end{abstract}

\title{
Table of Content:
}

Abstract

1 - Introduction

2 - Material and Methods

2.1 - The search for Schizophrenia Biomarkers: the description of the paradigms

2.1.1 - The Pilot Study in Munich

2.1.2 - The Pilot Study in Jülich

2.2 - The PET/MR/EEG trimodal instrument

2.2.1 - The MR scanner

2.2.2 - The PET scanner

2.2.3 - The EEG

2.2.4 - Integration of the three modalities

3 - Results

3.1 - Results of the Pilot study

3.1.1 - Recruitment and inclusion criteria

3.1.2 - Analysis of the Munich data

3.1.3 - Analysis of the Jülich data

3.2 - Preliminary results of the performances of the instrument

3.2.1 - MR performance

3.2.2 - PET performance

3.2.3 - Attenuation correction

4 - Discussion and Conclusions

5 - Vitae

6 - Acknowledgments

7 - References

8 - Supplemental files

8.1 - Analysis of the Jülich data

8.2 - Recruitment and inclusion criteria

8.3 - References for the supplemental files 


\section{Introduction}

The diagnosis of schizophrenia is usually performed clinically by an operational approach such as implemented in the two most widespread diagnostic systems ICD-10 and DSM-V. Schizophrenic disorder is primarily characterized by so-called "positive symptoms" such as delusions, hallucinations and thought disturbances, and "negative symptoms" such as blunted emotions, social withdrawal, catatonic behavior and lack of spontaneity [1]. In addition to positive and negative symptoms, cognitive deficits are widely considered a core feature of the disorder and play a role as vulnerability indicators, as enduring abnormality across psychotic and clinically remitted periods and have a strong predictive influence on functional recovery [2]. Cognitive impairments include symptoms of deficits in attention, memory and executive functions as well as social cognitive deficits such as constraints in emotion recognition, theory of mind and social attributional style $[3,4]$.

The dopamine system is the neurotransmitter system which has been most commonly associated with schizophrenia, under the hypothesis that schizophrenic patients undergo an increased dopamine synthesis capacity in the striatum as compared to healthy subjects [5]. While an enhanced activity within the subcortical mesolimbic dopaminergic pathway is associated with positive symptoms, negative symptoms and cognitive deficits are related to a hypoactive prefrontal mesocortical dopaminergic system [6-8]. Besides the widely discussed influence of dopaminergic neurotransmission, there is increasing evidence that alterations of serotonergic functioning also play a crucial role in the pathophysiology of negative symptoms in schizophrenia [9].

However, in healthy volunteers the N-methyl-D-aspartate (NMDA) receptor antagonist ketamine led to more effects like negative symptoms than the serotonin 5-HT2A agonist N, N-dimethyltryptamine (DMT) as a typical hallucinogen [10]. Meta-analyses have reported that NMDA receptor agonist Dserine and glycine transporter type 1 inhibitor sarcosine reduce total and negative symptoms as an adjunct to antipsychotics [11,12]. Based on these observations, it can be inferred that glutamate is also involved in the pathophysiology underlying negative and cognitive symptoms in schizophrenia. Newer approaches assume that the dysregulation of subcortical dopamine system functioning is secondary to hyperactivity within hippocampal subfields. Preclinical and human imaging studies suggest an activation of the ventral hippocampus secondary to a loss of interneuron function which leads to dopamine hyperfunction [13]. Results from animal and human studies indicate dysfunctional GABAergic inhibition [14]. Additionally, the glutamatergic system is involved in the development of the cognitive impairment. Repeated exposure to NMDA receptor antagonists leads to changes in the GABAergic markers that mimic the impairments found in schizophrenia $[13,15]$. From the above studies, it becomes clear that the different neurotransmitter systems (dopamine, serotonin, glutamate, GABA etc.) are closely related and cannot be considered separately.

While alterations in brain structure are robustly seen in schizophrenia with standard Magnetic Resonance (MR) techniques, they may be not diagnostic, in particular during the prodromal phase of the illness, and structural alterations may be common to patients with psychotic features across diagnostic boundaries [16]. Further, structural changes in the schizophrenic brain are likely to be the end point in the chain of pathological events that lead to symptoms. Unlike structural investigations of the brain, it has only been possible to study alterations in brain function with the advent of functional neuroimaging techniques. Positron Emission Tomography (PET) is a well-established imaging technique in the field of oncology, cardiology and neurology [17]. PET and blood oxygenation level dependent (BOLD)-based fMRI provide a reasonable balance of spatial and temporal resolution to study regional brain function. On the one hand, fMRI does not require ionizing radiation and rather relies on the magnetic properties of endogeneous haemoglobin in the brain. On the other hand, PET allows to address metabolism and neuroreceptors in a way that MRI simply cannot. In fact, whereas in BOLD-based fMRI studies, the detected signal is related to the haemodynamic responses accompanying neural activity, PET allows studying, at different stages, specific biochemical processes involved in the control of cerebral activity. This is possible thanks to 
the development of new radiotracers, such as dopaminergic (e.g., F-DOPA), gabaminergic and glutamatergic radiotracers, now available for research and for clinical applications.

Neurophysiological techniques involve the assessment of electrical activity of the brain using scalp electrodes at rest or while the subjects participate in a given experimental paradigm. Electroencephalography (EEG) is a routine approach in clinical psychiatry today. Especially evoked potential parameters such as mismatch negativity (MMN), P300 [18] and NoGo-Anteriorization [19] are being introduced into clinical routine due to their high sensitivity in detecting schizophrenic disorders. EEG is advantageous for its temporal resolution, far better than in other imaging methods, and for the time course analysis. The drawback is the relatively poor spatial information, .e.g. $1 \mathrm{~cm}$ in the cortex with evoked potentials [20]. Substantial research has been carried out to clarify the relationship between neurotransmission and evoked potentials in EEG. For example, the loudness dependence of auditory evoked potentials (LDAEP) is closely related to serotonergic neurotransmission and the mismatch negativity (MMN) to glutamatergic transmission [21,22].

In summary, PET, MR and EEG provide complementary anatomical, physiological, metabolic, and functional information about the brain. Pooling information obtained with each modality has long been performed through parallel analysis of the sequentially acquired data and, more commonly today, by using software co-registration techniques. The rationale for multimodal imaging in the form of simultaneous MR/EEG, and the extension thereof to triple modality PET/MR/EEG has been already discussed in the literature [23]. However, an off-line combination of the MRI and PET data acquired separately at different times is still insufficient because a number of correlated, patientspecific influences such as blood flow, breathing, pulse rate and other physiological parameters may vary over time and therefore potentially in different ways in the two scanners. As a consequence, the full integration of different diagnostic modalities into a seamless clinical tool is mandatory for the acquisition of multi-parametric measurements on a routine basis in schizophrenia patients.

In this paper, we describe the status of the TRIMAGE project funded by EU (www.trimage.eu) that started at the end of 2013 and is expected to be completed by the end of 2018. The TRIMAGE project aims to create a trimodal, simultaneous, cost-effective PET/MR/EEG imaging tool that makes use of cutting edge technology with performance beyond the state-of-the-art. By integration of three relevant modalities, it will facilitate multiparametric characterization of brain tissue in a single diagnostic session. Since these modalities are involved in early diagnosis of schizophrenia, the project TRIMAGE is on this case first. This involves not only the development of integrated hardware but also the whole chain of image generation and multimodal data analysis. Once established, it may also be applicable to other mental health disorders such as Tourette syndrome [24].

The TRIMAGE project [25] is coordinated by the Department of Physics of University of Pisa and is run by a consortium of 11 partners, seven from academia and four from the SME environment, namely: Department of Physics of Pisa University, Technological Educational Institute of Athens, INFN Torino, Technische Universitat Munich, Forschungszentrum Jülich GmbH, JARA BRAINRWTH Aachen, Department of Psychiatry, Psychotherapy and Psychosomatics, University of Zurich, AdvanSiD s.r.l., WeeROC s.a.s., raytest GmbH and RS2D s.a.s.

PET/MR instrumentation available today can be one of these three types [26]: sequential systems combine both modalities in the same manner as PET/CT systems do, placing them in a tandem configuration with some space between the two tomographs; a second approach relies on a removable PET insert, which can be placed within the bore of the MR scanner; finally, integrated systems include the PET detectors inside the MR scanner. The solution adopted for the TRIMAGE scanner is the fully integrated solution with the PET scanner positioned between the gradient coils and the RF coil, so as to optimize the space and the combined scanner performance. A schematic drawing of the proposed TRIMAGE scanner is presented in Figure 1. 
The main objectives of this project are two-fold: (a) to build and optimize an integrated diagnostic solution including a molecular imaging tool based on simultaneous PET, MR and EEG; (b) to validate the new tool with specific protocols for detecting characteristic patterns in asymptomatic and at-risk patients and monitoring medication during drug therapy. The project is directed towards a new, costeffective and integrated, "beyond the state-of-the-art" instrument in order to provide a toolset for the diagnosis of patients in most clinical psychiatric centres. The TRIMAGE project aims to discover and develop suitable biomarker combinations that are based on structural-functional-metabolic changes in the brain. The marker of functional changes will focus on alterations in the dopaminergic and the glutamatergic neurotransmitter systems using appropriate PET tracers. Thus, parallel to developing the combined instrument, TRIMAGE performs studies on existing PET/MR instrumentation available at partner institutions so that protocols and processing pipelines are optimized and can be implemented in the new device immediately after hardware development.

Here we report on the concept, status, and first results of this multi-faceted international project.

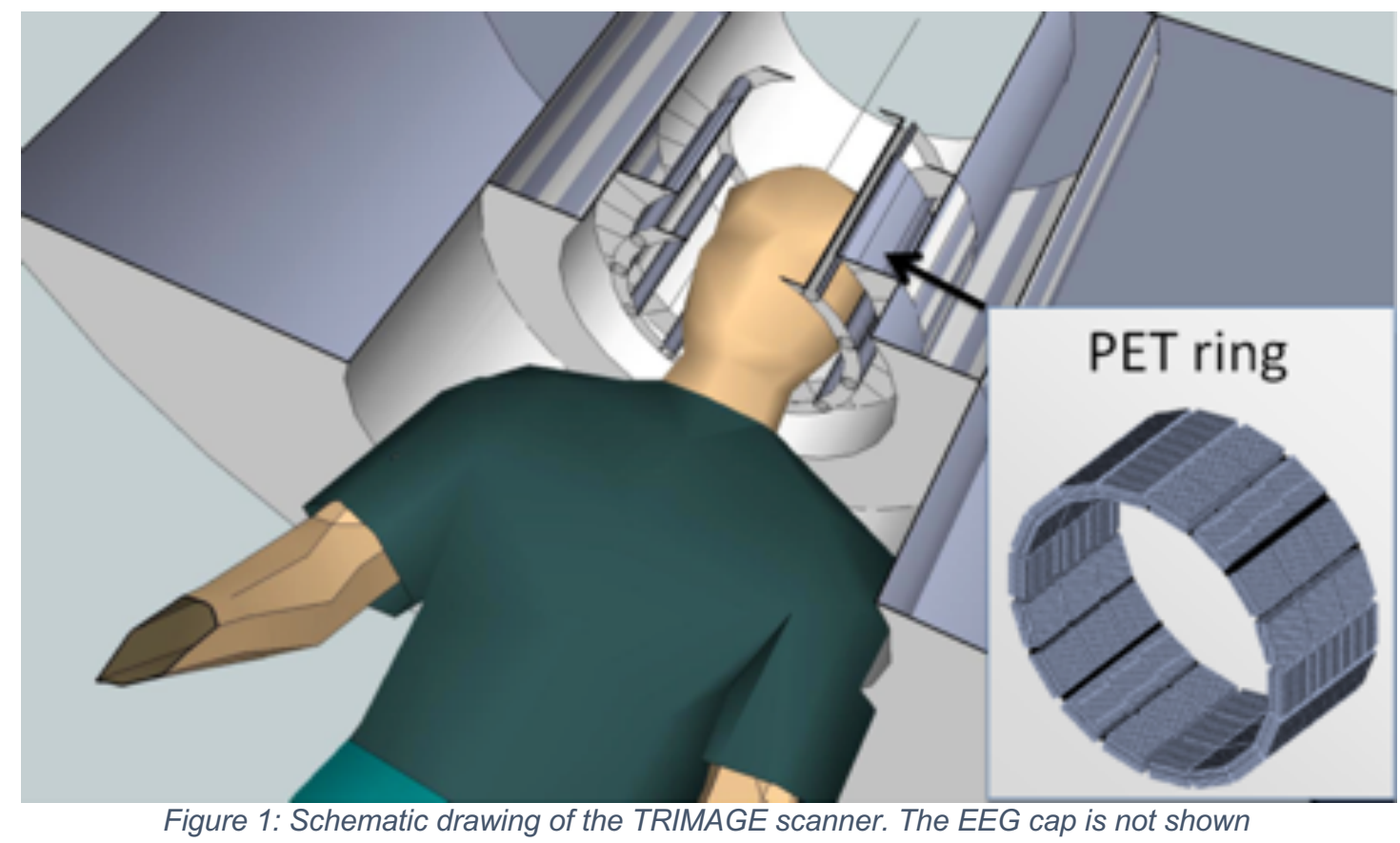

\section{Material and Methods}

\subsection{Imaging Schizophrenia}

At two partner sites, clinical PET/MR instrumentation is available that was used for imaging studies in the context of schizophrenia with the goal of adapting existing paradigms, so that they can be transferred to the TRIMAGE scanner. The MR-sequences were defined as compatible as possible in order to be able to perform post-hoc pooling and comparisons between the MRI data of the two sites. While dopaminergic imaging was the focus in Munich, glutamatergic studies are performed in Jülich, where EEG acquisition during PET/MR scans is an additional research focus.

\subsubsection{The Pilot Study in Munich}

Simultaneous $\left[{ }^{18} \mathrm{~F}\right]$-FDOPA PET and MRI was carried out in the combined whole-body mMR Biograph PET/MRI scanner (Siemens AG-Healthcare, Erlangen, Germany) [27]. [ $\left.{ }^{18} \mathrm{~F}\right]-\mathrm{FDOPA}$ is a radioactively labelled precursor of dopamine, that permits measurement of presynaptic dopamine synthesis in the brain. The goal was to optimize the protocol towards quantitative imaging with maximum patient compliance. 
PET scanning lasted $70 \mathrm{~min}$ and started 30 seconds before an intravenous injection of $150 \mathrm{MBq}$ of $\left[{ }^{18} \mathrm{~F}\right]$-FDOPA. PET data were reconstructed using ordered subset expectation maximization (OSEM) (21 subsets, 3 iterations) with a voxel size of $1.7 \times 1.7 \times 2 \mathrm{~mm}$ and corrected for attenuation and scatter based on anatomical MR information. 30 dynamic frames were created $(1 \times 30 \mathrm{~s}, 10 \times 15 \mathrm{~s}, 3 \times 20 \mathrm{~s}, 2 \times 60 \mathrm{~s}$, $2 \times 120 s, 12 \times 300 s$ ). The MRI sequences were acquired simultaneously with PET. Resting-state functional MRI data were obtained by three separate sequences: whole-brain, focus on basal ganglia and orbitofrontal cortex, and focus on medial temporal lobe. T1-weighted anatomical data were also acquired.

The protocol adopted at Munich is described in Table 1 and illustrated in Figure 2.

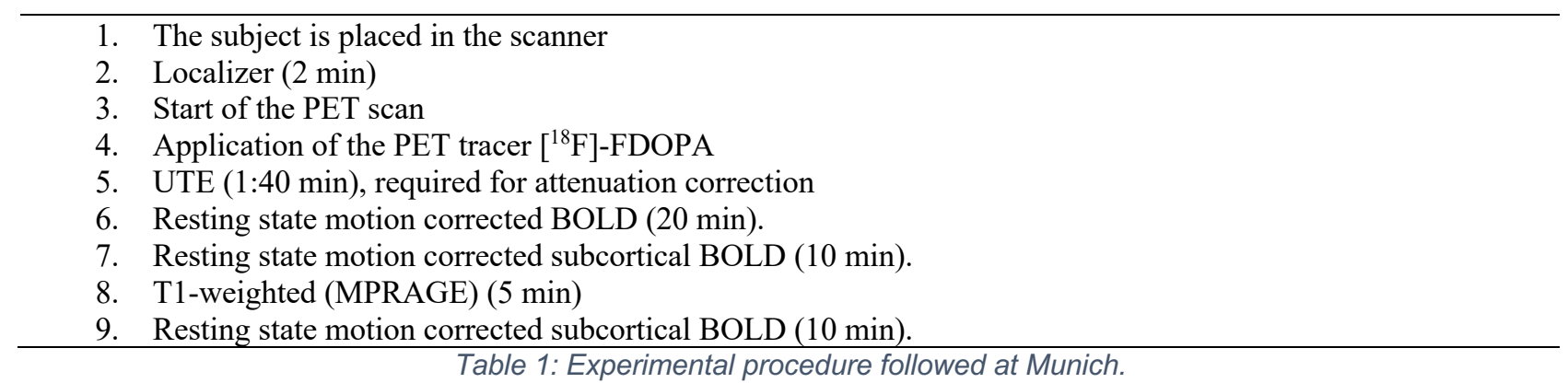

$0 \mathrm{~min} \quad 10 \mathrm{~min} \quad 20 \mathrm{~min} \quad 30 \mathrm{~min} \quad 40 \mathrm{~min} \quad 50 \mathrm{~min} \quad 60 \mathrm{~min} \quad 70 \mathrm{~min}$

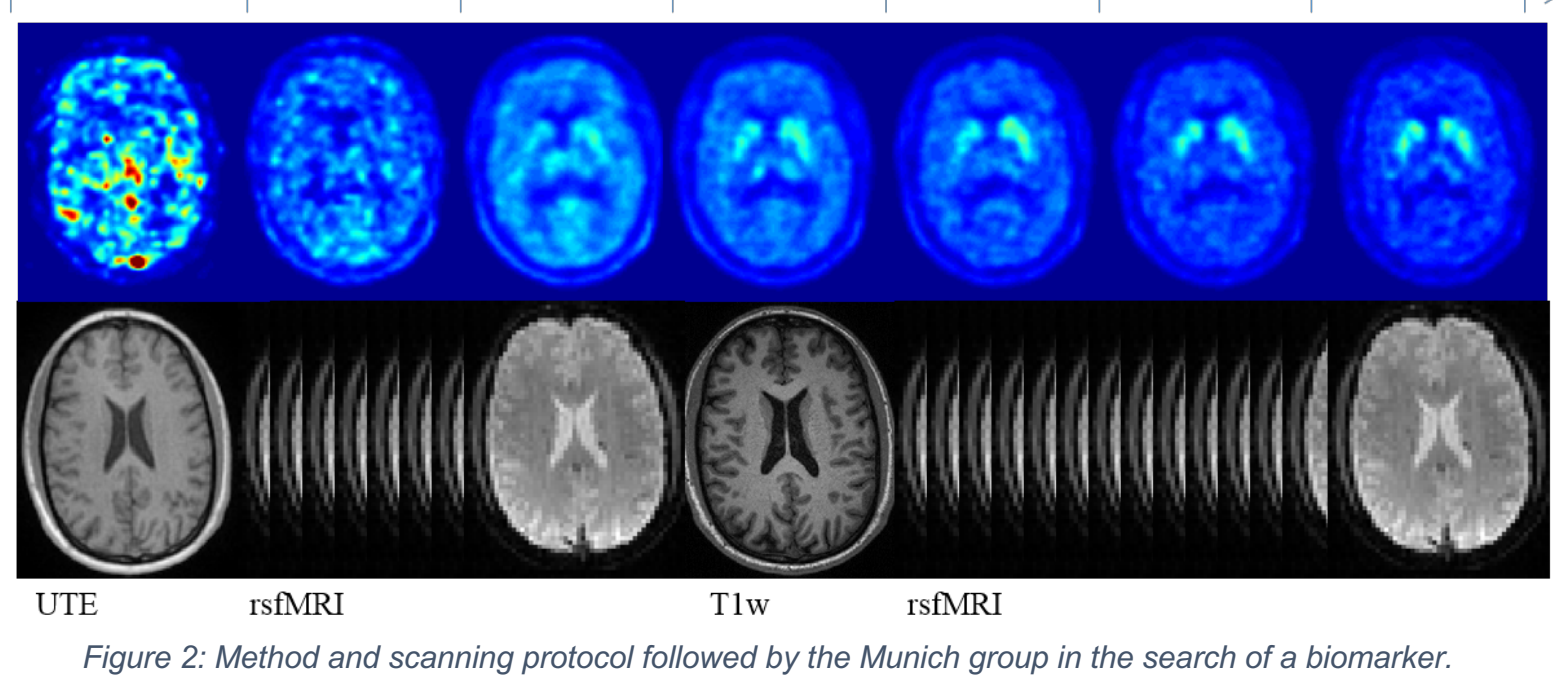

PET data were motion corrected by realigning all frames (starting from $5 \mathrm{~min}$ ) to the last PET frame (65-70 minutes), and then applying the transformation matrix for the last frame to all the early frames. A graphical Patlak analysis over the time frames from 20 to 60 min was conducted obtaining wholebrain kinetic parametric maps. From Patlak analysis the dopamine synthesis capacity can be measured through the index of the influx Kc. The simplified reference tissue model [28], with the cerebellum as reference, was adopted to circumvent the necessity of arterial blood sampling. To correct for FDOPA metabolites the signal from the cerebellum was taken as a surrogate of the metabolite measurement from the plasma [29].

\subsubsection{The Pilot study in Jülich}

A pilot study to explore the feasibility to record and to validate the trimodal acquisition protocol was designed and carried out on a 3T MR/BrainPET scanner (Siemens AG-Healthcare, Erlangen, Germany) equipped with a 64-Channel MR compatible EEG system (Brain Products, Germany). ${ }^{11} \mathrm{C}$ ABP688 tracer, which is a special ligand of the m-Glu5-receptor [30] was applied as PET tracer. A 
healthy male volunteer (age 26) as control and a schizophrenic patient (age 49) were included in this exploratory feasibility study.

The main objectives of this study were the following,

- To optimise and validate the PET $\left[{ }^{11} \mathrm{C}\right]-\mathrm{ABP} 688$ bolus + infusion scheme.

- To validate the MMN paradigm in EEG and fMRI for simultaneous acquisition.

- To perform an exploratory pilot analysis on the acquired trimodal data by calculating

$\circ$ the binding potential (BP) in target regions like the precuneus, cingulum posterior, hippocampus and parahippocampus, nucleus accumbens, frontal, mid- and inferior cortex and cerebellum using PET data,

- functional connectivity measures using fMRI data and

○ to investigate the event related potentials (ERP's) related to MMN in EEG data.

Since the pilot study in Jülich was conducted only to show the feasibility of simultaneous trimodal measurement using $\left[{ }^{11} \mathrm{C}\right]-\mathrm{ABP} 688$ as tracer (in healthy controls as well as in Schizophrenic patients), the possible effects due to age, education, ethnicity, and smoking status between healthy controls and schizophrenic patients are not taken in to account.

\section{Data acquisition}

The trimodal data acquisition protocol [31,32] is illustrated in Figure 3. PET data acquisition in list mode started simultaneously with the injection of the $\left[{ }^{11} \mathrm{C}\right]$-ABP688 tracer. Immediately afterward MRI (structural and functional - MMN task) and EEG (only during MMN task) data were recorded at common timepoints.
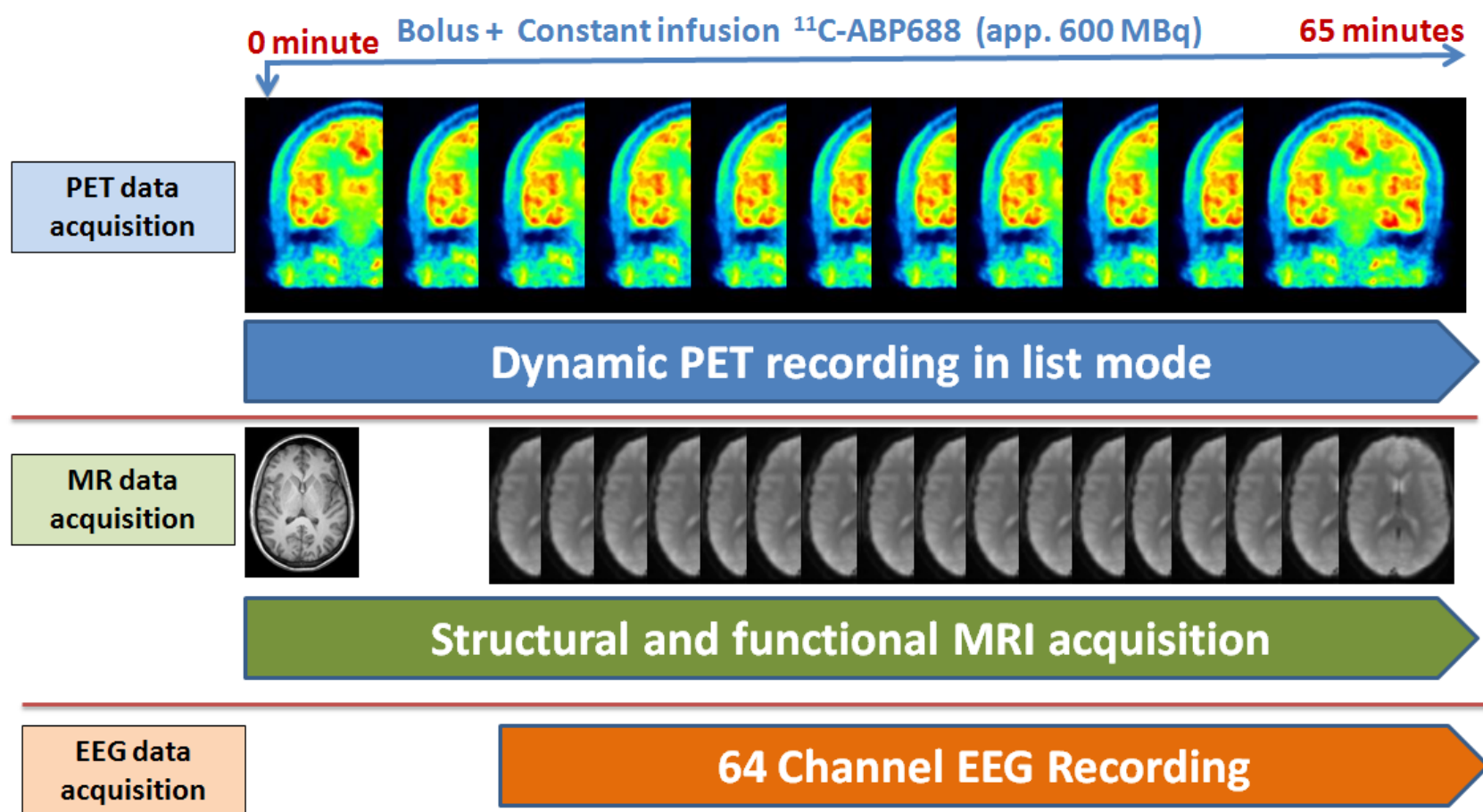

\section{Channel EEG Recording}

Figure 3: Trimodal data acquisition protocol developed and implemented by the Juelich group.

Description of the parameters applied in each imaging modality and MMN paradigm designed for this study are as follows.

\section{MMN Paradigm}

The MMN paradigm included changes in tone duration (standard tone $50 \mathrm{~ms}$, deviant tone $100 \mathrm{~ms}$ ). Auditory stimuli $(1 \mathrm{kHz}, 10 \mathrm{~ms}$ attack/decay) were presented in alternating sequences of mostly 50 ms standard tones with fewer $100 \mathrm{~ms}$ deviant tones in position 9 up to 16 after the standard tone (SOA $0.85 \mathrm{~s}+/-50 \mathrm{~ms}$ ). The deviant positions were pseudo-randomized (only two equal positions following each other). A total of 1410 trials ( $8 \%$ deviant, $92 \%$ standard) were presented. 


\section{MRI}

Anatomical images were acquired with MPRAGE sequence $(\mathrm{TR}=2250 \mathrm{~ms}$, TE $=3.03 \mathrm{~ms}, 176$ sagittal slices, $1 \mathrm{~mm}$ slice thickness, GRAPPA factor 2). A T2*-weighted EPI sequence (TR $=2.2 \mathrm{~s}$, $\mathrm{TE}=30 \mathrm{~ms}, \mathrm{FOV}=200 \mathrm{~mm}$, matrix size $64 \times 64$, slice thickness $=3 \mathrm{~mm}, 36$ slices) was used for the acquisition of the functional images ( 620 volumes).

\section{PET}

Patient and volunteer were injected with $575.2 \pm 0.57 \mathrm{MBq}$ of ${ }^{11} \mathrm{C}-\mathrm{ABP} 688$ via bolus injection plus constant infusion during 65 minutes [33] with 53 minutes of bolus magnitude component as already validated by other groups [34]. Equilibrium between plasma and tissue was noticed at 30 minutes.

Data were acquired in list mode, iteratively reconstructed with 3D OSEM 32 iterations and 2 subsets: 42 frames, 30 frames with $10 \mathrm{~s}$ frame length, 6 frames with $220 \mathrm{~s}$ frame length (frames between the task intervals settings were not considered in the analysis), matrix size $256 \times 256,153$ slices, voxel size $1.25 \mathrm{~mm}^{3}$ isotropic. MR-template-based attenuation correction [35] was applied. The AC method adopted by Jülich was different from the one by Munich on a different scanner. However, a comparison between the two AC methods showed compatible performance [36]. PET data were corrected for decay, dead time, random events and scatter.

\section{EEG}

EEG signals were recorded simultaneously during the MMN task paradigm, along with fMRI and PET data, using a commercially available MR-compatible EEG-system (Brain Products, Germany). EEG signals were recorded relative to the channel Fpz as reference and a ground electrode was located at AFz (10-5 electrode system). Signals were sampled at $5000 \mathrm{~Hz}$, with a bandwidth between 0.016 and $250 \mathrm{~Hz}$. During the measurement, the subject was requested to lie down in a supine position and relax. A silent "Mr. Bean" video was presented to the subject. The helium pump of the magnet was switched off during simultaneous measurement.

\section{Data analysis of the Juelich data}

A detailed description of the data analysis of the Juelich data is presented in the supplemental files.

\subsection{The PET/MR/EEG trimodal instrument}

\subsubsection{The MR scanner}

The MR scanner is designed to be fully compatible with the PET module. The magnet integrates a new technology bringing together a very compact design with a cryogen-free magnet at the most widely available clinical field of $1.5 \mathrm{~T}$. The limited axial dimension reduces the claustrophobic effects for the patient and gives the possibility of PET bolus injection under control, the arms outside of the magnet. Figure 4 represents the actual dimension of the magnet produced by SSI, Superconducting Systems Inc., U.S.A. The magnet coil is cooled with a cryocooler that takes a minimum amount of space near the magnet and allows the system to work without any cryogenic fluids (no liquid helium, no liquid nitrogen). Eighteen days are needed to cool down the magnet before energizing it. As a result, the simpler safety requirements reduce the cost and the installation complexity.

The magnet has been temporarily installed at RS2D (Strasbourg, France) for testing. A dedicated Faraday cage was built and installed around it. A description of the general properties of the MR component for the parts in the operating room and in the technical room is given in Table 2.

In addition to the measurement of the static $\mathrm{B}_{0}$ and of the magnetic field gradient strengths, imaging measurements were carried out on a standard MR phantom consisting of a PMMA cube (135 x 135 x $135 \mathrm{~mm}^{3}$ ) inside a cylinder (200 $\mathrm{mm}$ diameter and $160 \mathrm{~mm}$ length) filled with a preparation composed of 51 of demineralized water, $18 \mathrm{~g}$ of $\mathrm{NaCl}$ and $6.25 \mathrm{~g}$ of $\mathrm{CuSO}_{4}$.

The selected RF coil is designed in two layers: the outer layer is a dedicated transmit-only, quadrature birdcage coil and the inner part is a receive-only, 8-channel phased array coil. Since the delivery date for this coil is December 2017, two other coils were used for testing the sequences to be implemented on the MR Scanner. The second one is an eight-channel degenerate birdcage coil for transmission and detection, manufactured using non-magnetic, fixed and variable capacitors located outside the 
PET field-of-view. The coils support frame is 3D printed on a polycarbonate substrate. The third coil is a quadrature birdcage coil built with copper tape on a PMMA cylinder tuned for the 1.5T magnet.

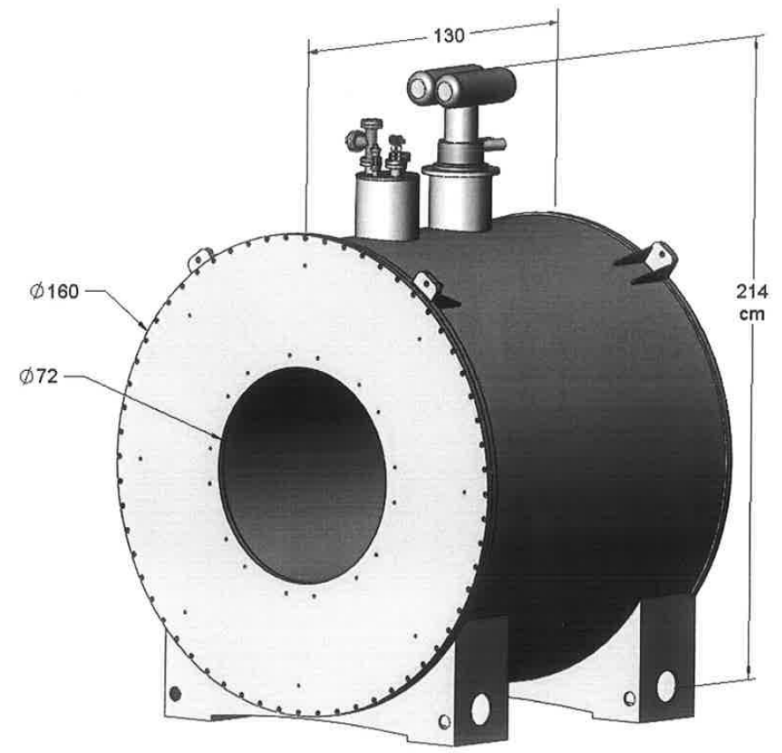

Figure 4: Schematic drawing of the MR magnet.

\begin{tabular}{|c|c|}
\hline \multicolumn{2}{|l|}{ Operating room } \\
\hline Magnet & Main horizontal magnetic field $\mathrm{B}_{0}$ at $1.5 \mathrm{~T}$ \\
\hline Gradient coils & $\begin{array}{l}\text { Space encoding gradients along the three directions } \mathrm{X}, \mathrm{Y} \text { and } \\
\mathrm{Z} \text {. Shims coils for field homogeneity correction. }\end{array}$ \\
\hline Ferro shim & For passive shimming \\
\hline $\begin{array}{c}\text { RF coil } \\
\text { Technical room }\end{array}$ & Transmission of the $\mathrm{B}_{1}$ field and reception of the MR signal \\
\hline Cryocooler & Water-cooled helium gas compressor for the magnet cooling \\
\hline Gradient amplifier unit & $\begin{array}{l}\text { Gradient current amplifier and power supply for the gradient } \\
\text { pulse generation }\end{array}$ \\
\hline Cameleon electronics & Acquisition electronics (spectrometer) \\
\hline RF Amplifier & $\begin{array}{l}\text { For the high voltage generation needed by the RF coil to } \\
\text { generate } B_{1} \text { field }\end{array}$ \\
\hline RF Interface box & $\begin{array}{l}\text { Electronics including coil identification, SAR control, active } \\
\text { decoupling, Transmit/Receive switch }\end{array}$ \\
\hline Shim power supply & Shim current amplifier \\
\hline Monitoring unit & $\begin{array}{l}\text { For the gradient monitoring and system protection } \\
\text { Table 2: MR System components. }\end{array}$ \\
\hline
\end{tabular}

\subsubsection{The PET Scanner}

The PET component of the TRIMAGE system features a full ring (Figure 5, right) comprising 18 $\mathrm{LYSO} / \mathrm{SiPM}$ rectangular detectors [37], $55 \mathrm{~mm}$ (transversal) $\times 163 \mathrm{~mm}$ (axial), with each one consisting of three square detector modules hosted in an RF shielded cassette. Each module is divided into four sub-modules, which we refer to as "tiles" (Figure 5, left). A total of 216 tiles forms a full ring of $31 \mathrm{~cm}$ inner diameter. Each tile comprises two segmented LYSO crystal layers. The top layer (the nearer to the center of the field-of-view) consists of $7 \times 7$ crystals of $3.3 \times 3.3 \times 8 \mathrm{~mm}^{3}$, while the bottom layer has $8 \times 8$ crystals of $3.3 \times 3.3 \times 12 \mathrm{~mm}^{3}$. Both layers have a pitch of $3.4 \mathrm{~mm}$ and are half-pitch "staggered", i.e., each crystal of the top layer is coupled to four crystals of the bottom layer. This configuration allows performing a dichotomic depth of interaction reconstruction, as photons interacting in different layers are expected to produce different light patterns on the SiPMs [38]. 


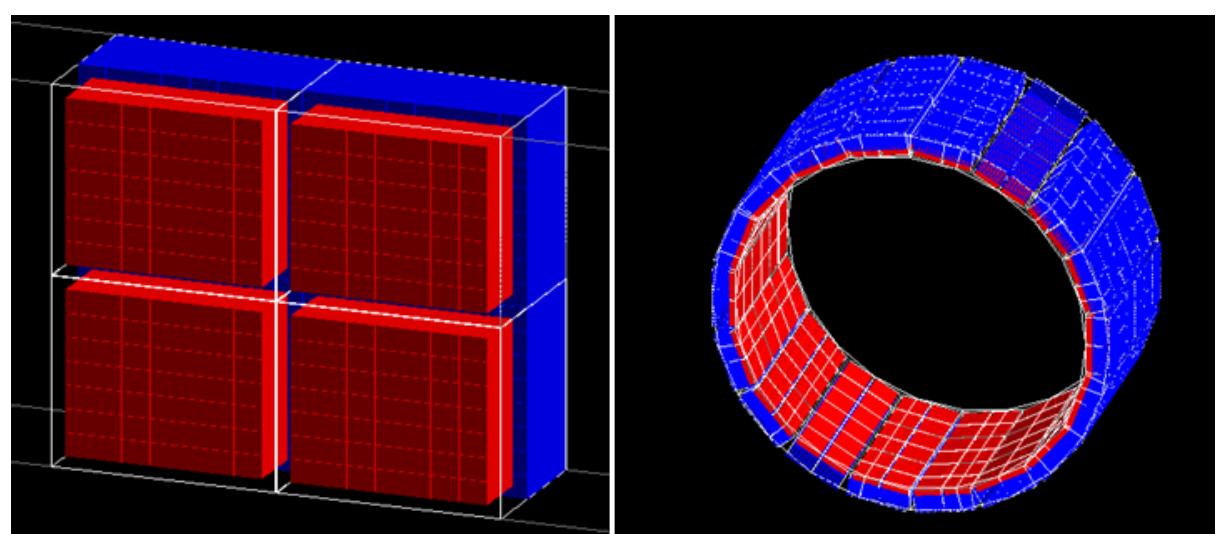

Figure 5: Left: module of the system (1st layer in red and 2nd layer in blue), Right: scheme of the full TRIMAGE scanner.

The crystals in the bottom layer are coupled one-to-one to 64 SiPMs that are arranged in two matrices of $8 \times 4$ NUV SiPMs with a size of $3 \times 3 \mathrm{~mm}^{2}$ manufactured by Advansid (Trento, Italy). All the 32 SiPMs are mounted on a common package which is completely covered with transparent epoxy layer. Each SiPM has approximately 5600 micro-cells, $40 \mu \mathrm{m}$ side with a $60 \%$ fill-factor.

The light emitted by each LYSO matrix and sensed by the SiPMs is read out by a 64-channel TRIROC ASIC [39]. Four TRIROC ASICs are hosted on a single front-end board (ASIC board) that is able to read out all the 256 output signals from a detector module. The digital part of the TRIROC ASIC manages the conversion and the data transmission to a FPGA-based board that is hosted inside the cassette, called TX board as it transmits the acquired data to the back-end for coincidence processing. The backend system is composed of a motherboard and 9 boards (RX boards) receiving data from two TX board. The FPGA of the motherboard multiplexes the data coming from the RX boards; it handles the slow control and sorts all the single events by timestamp. Sorted events are then processed in real time for coincidence detection by timestamp comparison.

Several fully assembled prototypes of TX boards have been tested for coincidence read-out. Board testing coverage included: slow control registers read/write, high-speed data transfer, clock distribution, on-chip memory storage, on-chip events processing [40].

\subsubsection{The EEG}

A commercially-available, 64-channel MR compatible EEG by Brain Products, Germany will be used. An explorative study investigating and quantifying the effect of the components of an MRcompatible EEG cap during a simultaneous trimodal study on PET images has just been completed in Juelich [24,32]. The study was designed to investigate the influence of the EEG cap on the quality and quantification of PET images acquired during simultaneous measurements in the Jülich brain PET/MR device. The Brain-PET-MR emission images of the Iida phantom with $\left[{ }^{18} \mathrm{~F}\right]-$ Fluorodeoxyglucose as well as of human subjects with EEG cap did not show significant artefacts caused by the EEG cap even though the applied attenuation correction did not take into account the attenuation of the EEG cap itself.

\subsubsection{Integration of the three modalities}

One of the main challenges in the design of the TRIMAGE hybrid scanner is to avoid interferences between components and parts of the PET and MR devices.

MR scanners, e.g., 1.5T MR scanner of the TRIMAGE project, use a number of radio frequency (RF) coils tuned to the Larmor frequency at $63.8 \mathrm{MHz}$ to generate a magnetic field $\mathrm{B}_{1}$, perpendicular to the static $\mathrm{B}_{0}$-field, which can inhibit the PET electronics during the data acquisition. On the other hand, the emitted noise from the PET electronics can interfere with the MR signal, which is in the $\mu \mathrm{V}$ range and is picked up by sensitive RF receiver coils.

To avoid these interferences, usually a shield is installed between the two parts. For the TRIMAGE scanner the shield is based on a thin metal layer with high conductivity (e.g., copper), which provides a high shielding effectiveness (SE). The shield should not interact with the static $\mathrm{B}_{0}$ or the gradient fields. The interaction with the static field has been avoided by considering non-magnetic, e.g., 
nickel-free metals, with relative magnetic permeability $\mu_{\mathrm{r}} \approx 1$, which do not perturb the homogeneity of the static field $\mathrm{B}_{0}$. The interaction between the shield and the gradients is more critical and has been carefully addressed. The main drawback of a standard shield based on a solid/continuous metal layer is the effect of eddy currents induced by the switching of gradient coils due to the law of induction. The fields produced by the eddy currents interfere with the gradient fields and change the encoding of the MR image. This effect is especially critical in MR sequences such as EPI and EPIK, which require fast switching gradients. In order to overcome this limitation, in the TRIMAGE project the standard solid metal shield has been replaced by an innovative frequency-selective shielding concept. The proposed solution is almost transparent to the gradient fields, thereby suppressing the eddy-current generation, while providing a high SE and EM screen for the PET electronics at the Larmor frequency. The frequency selective behaviour of the proposed shielding concept has been characterized via both simulations and measurements, including MR scans [41].

Another possible source of interference is via the distortion of $\mathrm{B}_{0}$ and $\mathrm{B}_{1}$ fields due to the presence of material with a high magnetic susceptibility such as iron or nickel. While it is relatively easy to avoid iron in PET components, a full nickel-free construction is more challenging. In fact, nickel is widespread use in the plating process of electrical connectors. For that reason, we have selected a custom Nickel-Phosphide plating (that has a negligible magnetic susceptibility) for the components of the PET electronics and for the board to board connectors.

Another interference to be considered is the one between the cryocooler frequency and the MR. This effect has also been studied and will be avoided by filtering out the cryocooler frequencies from the MR data.

\section{Results}

\subsection{Results of the pilot study}

\subsubsection{Recruitment and inclusion criteria}

Recruitment of subjects with schizophrenia diagnosis according to ICD-10 and age- and educationmatched healthy controls is described in the supplemental files. The recruitment took place at the two sites Munich and Jülich. Inclusion criteria were identical for both study sites.

\subsubsection{Analysis of the Munich data}

Presently, 12 patients with schizophrenia (mean age: $46.33 \pm 10.52$ years; 5 female) and 13 healthy control subjects (mean age: $43.76 \pm 12.26$ years; 6 female) were included in the study. All participants provided informed consent in accordance with the Human Research Committee guidelines of the Klinikum rechts der Isar, Technische Universität München.

Patients were recruited from the Department of Psychiatry by treating psychiatrists, healthy control subjects from the Munich area by word-of-mouth advertising. Participants' examination included medical history, psychiatric interview, and psychometric assessment. Included patients suffered from chronic schizophrenia (at least 2 psychotic episodes) and were currently in psychotic remission. The mean total PANSS score was 47. Eleven patients were taking antipsychotic drugs at the time of measurement.

A two-sample t-test was used in order to test whether the groups differed with regard to age. There was no significant statistical difference with regard to age between patients with schizophrenia (mean age 46.33 years, standard deviation 10.52) and healthy controls (mean age 43.76 years, standard deviation 12.26), $\mathrm{p}=0.58$. With regard to gender, a chi-squared test was used to test for the frequency of men ( 7 in each group) and women ( 6 in the healthy control group and 5 in the patient group) between the two groups, resulting in no significant interaction $(\mathrm{p}=0.83)$.

Figure 6 shows an exemplar Kc map together with the last PET frame (65-70 mins) of the same subject, both overlaid on the T1w for anatomical correspondence. In the PET frame, we see that most of the FDOPA is located in the striatum, together with some peripheral radio-metabolites. In the parametric map, a large number of voxels contained negative Kc values suggesting the unsuitability 
of the Patlak model for those voxels. Furthermore, the quality of the fitting $\left(\mathrm{R}^{2}\right)$ was measured for each voxel, showing that most voxels in the brain have a poor fitting quality, mainly excluding the striatum. Only those voxels with a high fitting quality $\left(\mathrm{R}^{2}>0.8\right)$ were considered in the region-ofinterest analysis.

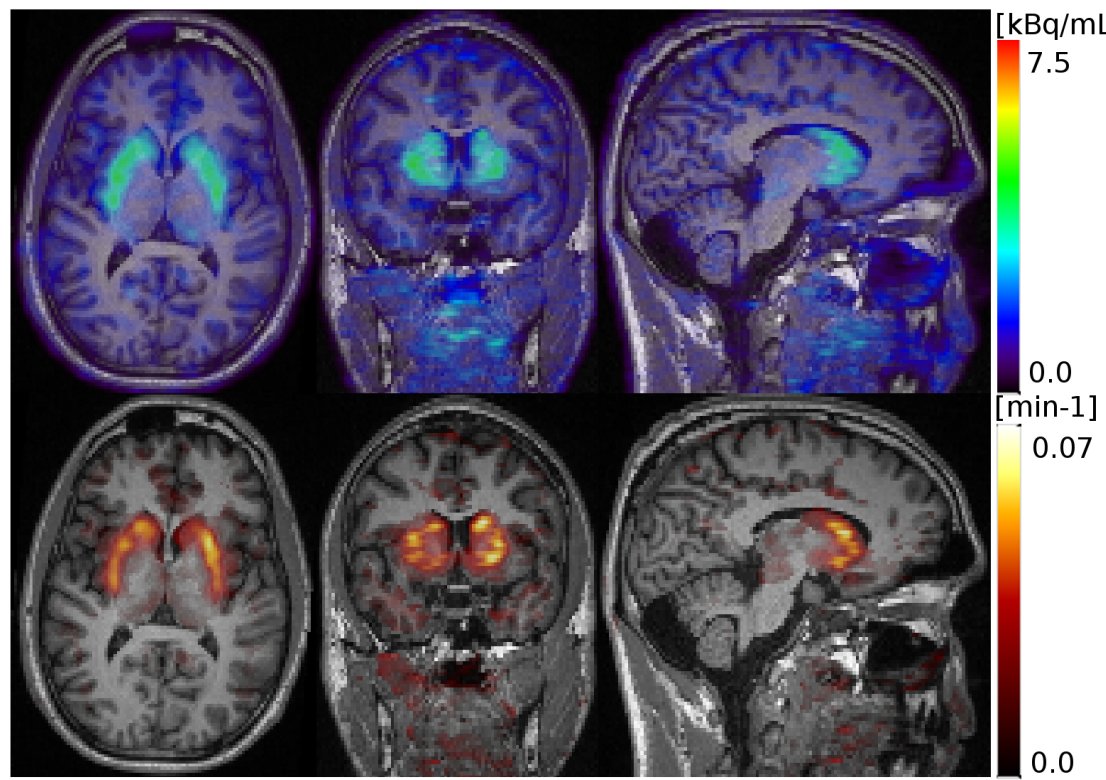

Figure 6: Axial, coronal and sagittal PET slices from the last frame (65-70 minutes) (top) and from the Kc parametric map (bottom) at the level of the striatum.

Anatomical masks of striatal subregions from the Hammersmith atlas (accumbens, caudate and putamen), and functional masks from the Oxford-GSK-Imanova connectivity atlas (limbic, sensorimotor and executive) were non-linearly co-registered to the PET images using the Statistical Parametric Mapping ${ }^{1}$ (v12) tool in Matlab R2016b (The Mathworks, Inc., Natick, Massachusetts, United States) and the anatomical T1-weighetd MRI data. Regional Kc values were extracted from all voxels within the mask and two-sided t-tests were conducted to investigate group differences. Schizophrenic patients showed increased Kc values in the nucleus accumbens $\left(0.040 \pm 0.005 \mathrm{~min}^{-1}\right)$ compared to healthy controls $(0.035 \pm 0.006 \mathrm{~min}-1)$ with statistical significance $(p=0.04)$ (Figure 7 centre).

Similarly, an increase of Kc values was found in the functional limbic region (Figure 7, right), which largely overlaps with the anatomical region of the accumbens, for schizophrenic patients $(0.039 \pm 0.006 \mathrm{~min}-1)$ compared to controls $\left(0.036 \pm 0.006 \mathrm{~min}^{-1}\right)(\mathrm{p}=0.043)$. No important differences were observed in the whole striatum (Figure 7, left) or other striatal sub-regions.
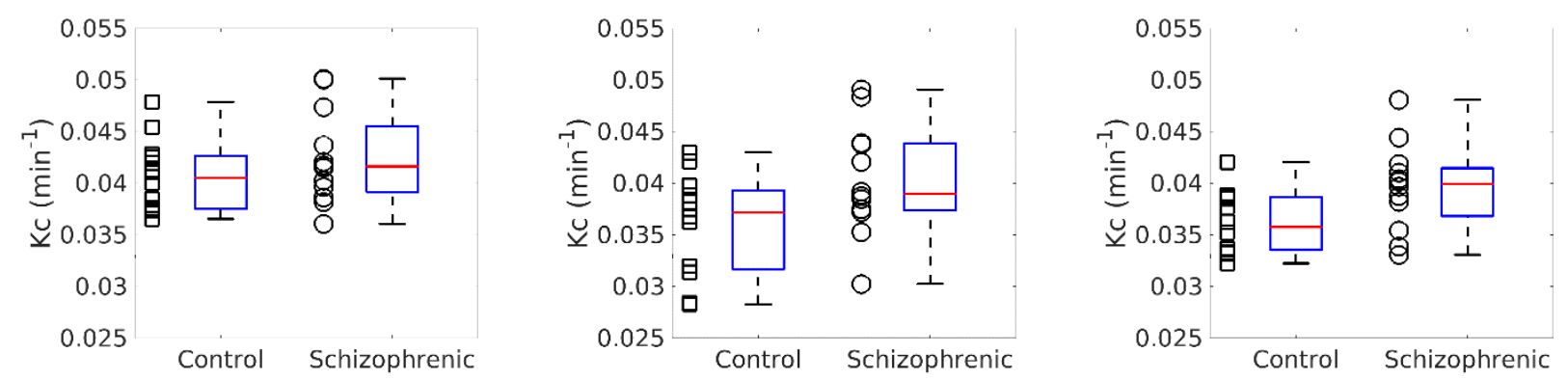

Figure 7: The index of influx, Kc, in the whole striatum (left), nucleus accumbens (centre) and limbic region (right) of the striatum as measured with the PET/MR (mMR) at Munich.

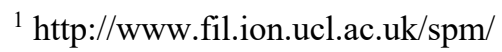




\subsubsection{Analysis of the Jülich data}

\section{FMRI}

The functional connectivity measures calculated for the healthy subject and the schizophrenic patient are shown in Figure 8. A two-sample t-test between GM mask and auditory network (AN) showed a significant difference $(\mathrm{p}<0.0001)$ in both healthy subject and schizophrenic patient. These data based on two subjects must be taken as an indication only; larger group sizes are required before any definitive statement can be made.

Similarly, the two-sample t-test between healthy subject and schizophrenic patient in AN and SN showed significant difference $(\mathrm{p}<0.0001)$. The short range functional connectivity measure, called regional homogeneity ( $\mathrm{ReHo}$ ), and the long range functional connectivity measure, called degree centrality (DC), both showed less activation in comparison to the healthy subject.

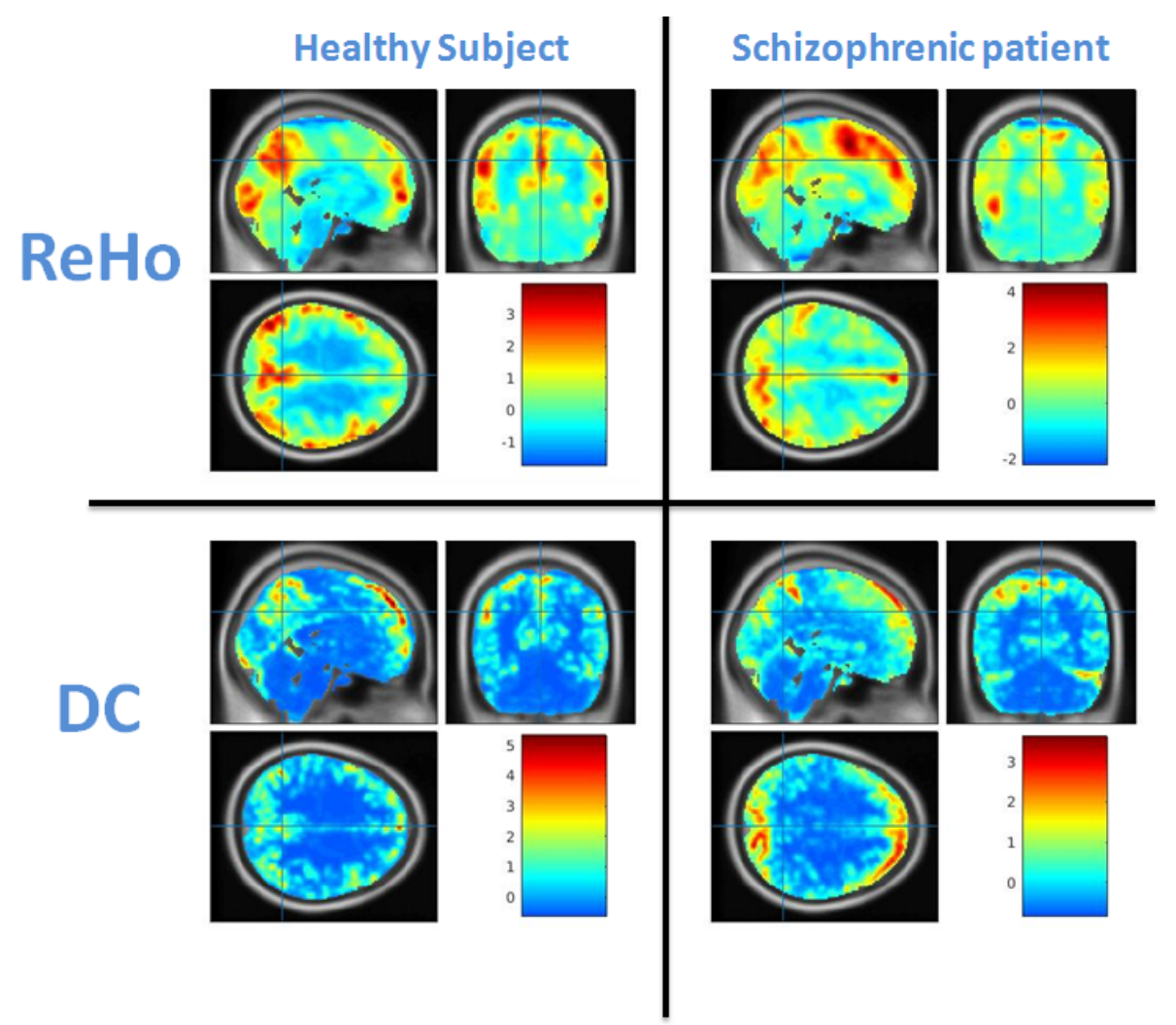

Figure 8: Functional connectivity measures, ReHo (top row) and DC (bottom row), for the healthy subject (right) and the schizophrenic patient (left). The colour bar shows $Z$ standardized values in all images.

\section{PET}

Time activity curves are shown in Figure 9 for the healthy control and the schizophrenic patient. The time activity curves of Figures 9 (top) and 9 (middle) show that we were in the equilibrium condition for non-displaceable Binding Potential calculations during the task. Average $\mathrm{BP}_{\mathrm{ND}}$ values during the MMN task are given in Table 3. Cerebellum was used as the reference region:

$$
\mathrm{BP}_{\mathrm{ND}}=\left(\mathrm{C}_{\text {region }}-\mathrm{C}_{\text {cereb }}\right) / \mathrm{C}_{\text {cereb }}
$$

Furthermore, to address differences in activity concentrations in this patient versus healthy volunteer we normalized it by the reference region and made a performed a t-test. As an example, the Cingulum Posterior region normalized by the activity concentration in the cerebellum is plotted in Figure 9 (bottom).

The deviations in $\mathrm{BP}_{\mathrm{ND}}$ represents the standard deviation between frames during the MMN task after 30 minutes. 

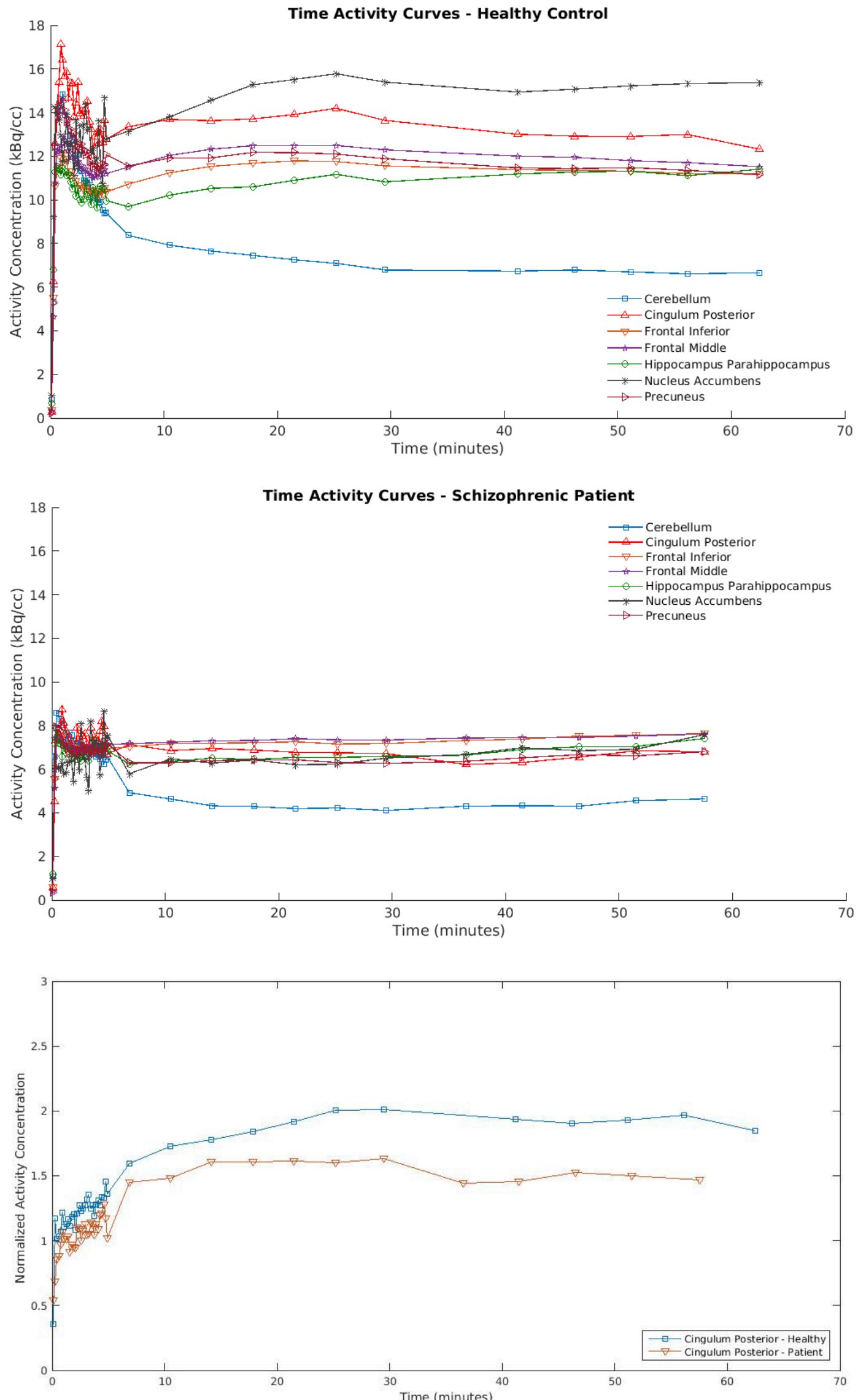

Figure 9: Time activity curves of the healthy control (top) and the schizophrenic patient (middle); normalized activity curve of the Cingulum Posterior for the healthy control subject and the schizophrenic patient (bottom). Equilibrium is considered at 30 minutes after bolus plus infusion started. 


\begin{tabular}{|c|c|c|}
\hline Region & BP $_{\text {ND }}$ Healthy Control & BP $_{\text {ND }}$ Schizophrenic Patient \\
\hline Precuneus & $0.70 \pm 0.02$ & $0.52 \pm 0.04$ \\
\hline Cingulum Posterior & $0.93 \pm 0.03$ & $0.50 \pm 0.04$ \\
\hline $\begin{array}{c}\text { Hippocampus and } \\
\text { Parahippocampus }\end{array}$ & $0.67 \pm 0.01$ & $0.61 \pm 0.05$ \\
\hline Nucleus Accumbens & $1.26 \pm 0.05$ & $0.64 \pm 0.05$ \\
\hline Frontal Cortex Inferior & $0.69 \pm 0.01$ & $0.74 \pm 0.04$ \\
\hline Frontal Cortex Middle & $0.77 \pm 0.01$ & $0.74 \pm 0.04$ \\
\hline
\end{tabular}

Table 3: Binding potential non-displaceable during task in different brain target regions.

\section{EEG}

The loudness dependence of auditory evoked potentials (LDAEP) during standard and deviant tone for control and schizophrenic patient are shown in Figure 10. The computed amplitudes for the healthy control and the schizophrenic patient are 2.38 and $1.25 \mu \mathrm{V}$, respectively.
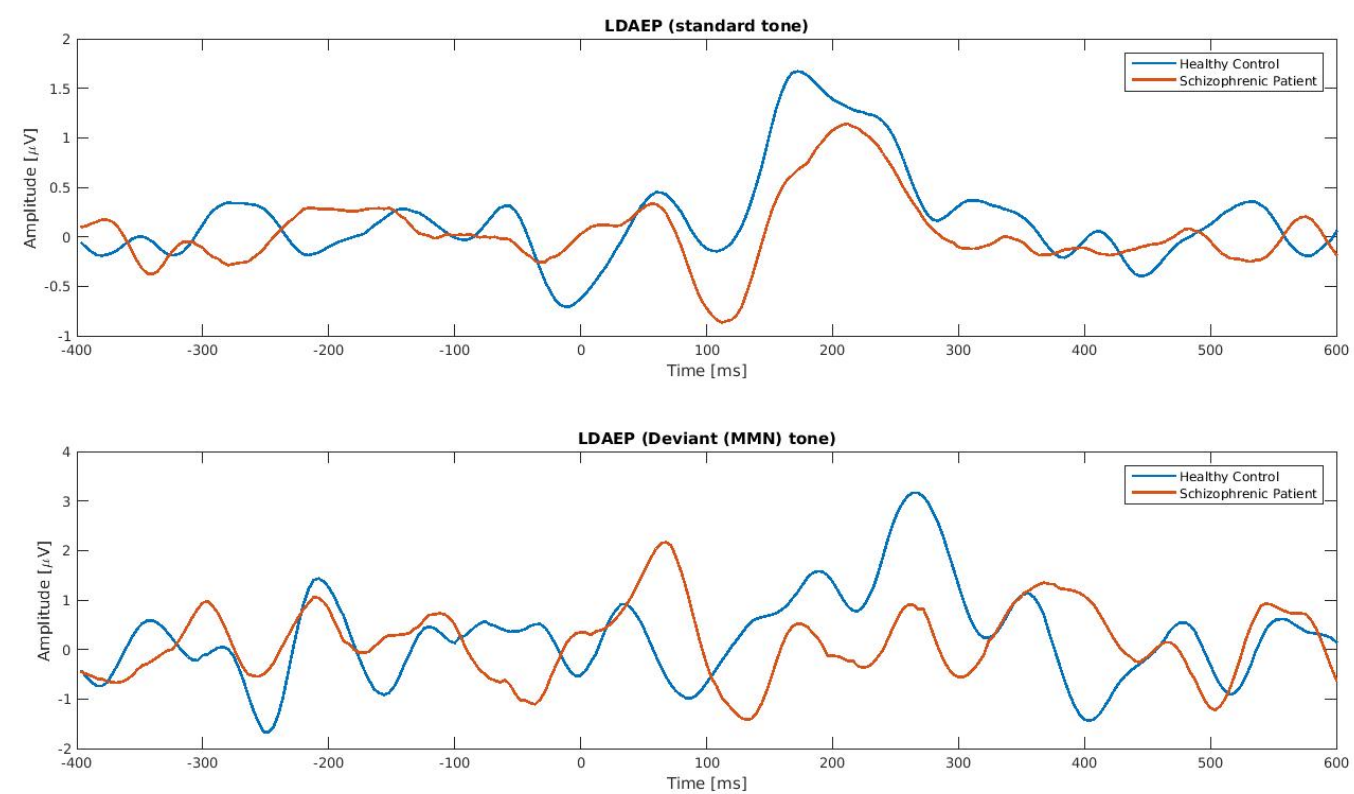

Figure 10: LDAEP during standard (upper row) and deviant tone (lower row). A reduction in amplitude of MMN (deviant tone, lower row) is evident between healthy subject and schizophrenic patient.

\subsection{Preliminary results of the performance of the trimodal instrument}

\subsubsection{MR performance}

The major properties and static measurements performed on the magnet are reported in Table 4.

To measure the performance of the gradient system a gradient echo profile sequence on the axis $\mathrm{X}$, $\mathrm{Y}$ and $\mathrm{Z}$ at maximum power was set up and the max voltage and the current monitoring port of the gradient amplifier were measured. The actual measured strength of the gradient was $41.23 \mathrm{mT} / \mathrm{m}$, $41.3 \mathrm{mT} / \mathrm{m}$ and $41.2 \mathrm{mT} / \mathrm{m}$ for the $\mathrm{X}, \mathrm{Y}$ and $\mathrm{Z}$ axis, respectively.

The gradient distortion measured with the PMMA phantom gave a standard deviation of the measured length of the phantom cube side of $0.69 \%, 0.75 \%$ and $0.93 \%$ for the $\mathrm{X}, \mathrm{Y}$ and $\mathrm{Z}$ axis, respectively. 


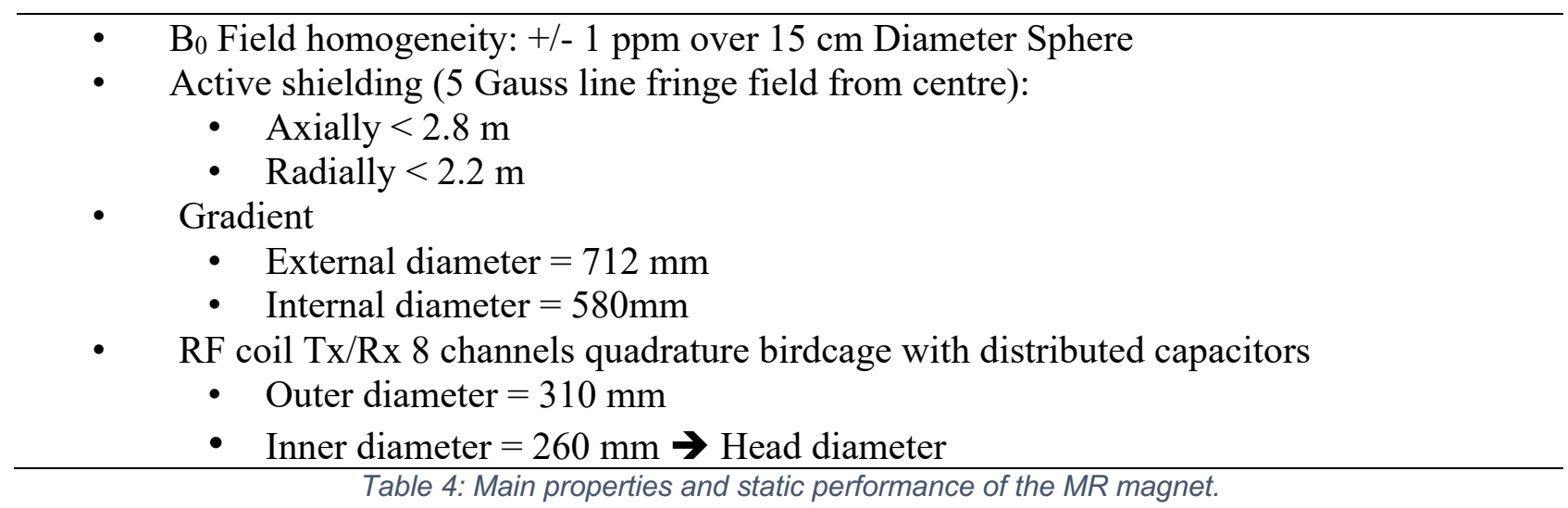

\subsubsection{PET performance}

Results on a demonstrator composed of a detector module readout by four TRIROC ASICs via a dedicated test bench are reported here. The flood map obtained with only the single layer shows well separated pixel spots indicating a proper ASIC and data acquisition functioning. The centroid algorithm implemented in the FPGA is also running to specifications with a $90 \%$ accuracy in pixel identification $[38,40]$.

The energy resolution at $511 \mathrm{keV}$ is $16 \%$ for the top layer and $18 \%$ for the bottom one. The worse energy resolution of the bottom layer can be attributed to the higher light dispersion that occurs when the scintillation light is produced in the bottom layer.

The best coincidence time resolution (CTR) obtained with two crystal pixels is shown in Figure 11. The obtained value $(420 \pm 33) \mathrm{ps}$ is at the state of the art of the advanced time-of-flight PET tomographs.

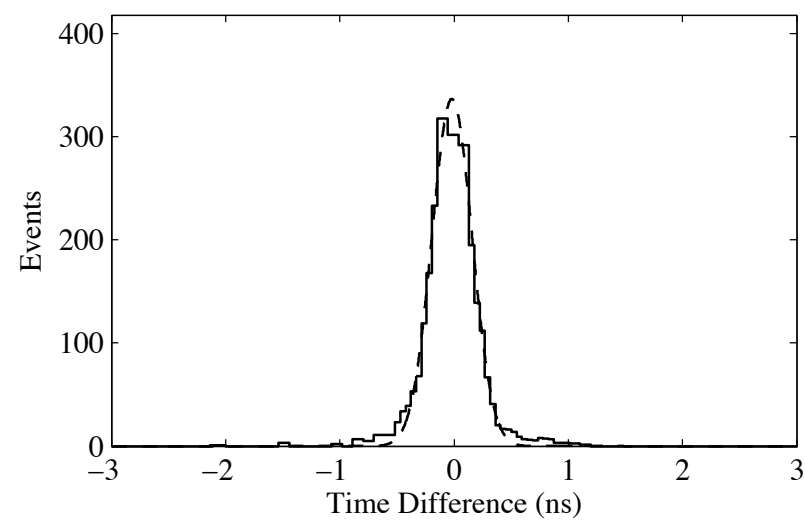

Figure 11: Coincidence time resolution (CTR) obtained with two crystal pixels. The CTR FWHM is (420 \pm 33$)$ ps.

\section{Simulation}

Monte Carlo simulations were established to study the expected system performance and generate data which dedicated image reconstruction algorithms can be developed with even before real data are available. The GATE Monte Carlo toolkit [42] was used for simulating the data using a precise description of the hardware under development. Several geometrical phantoms were implemented covering realistic imaging situations. For PET image reconstruction, the statistical iterative Maximum-Likelihood Expectation-Maximization (ML-EM) algorithm based on the PRESTO toolkit [43] was used, which was developed in Jülich for the MR-compatible BrainPET insert [44].

The simulated PET model was evaluated for its performance according to the NEMA standards. Specifically, the i) spatial resolution, ii) sensitivity and iii) count rate were evaluated on the basis of Monte Carlo simulations. For the lack of specific NEMA procedures for dedicated brain systems, NEMA standards [45-47] have been adapted case by case. 


\section{Spatial resolution}

An ${ }^{18} \mathrm{~F}$ back-to-back point source was used to assess the spatial resolution with $1 \mathrm{MBq}$ for $700 \mathrm{~s}$ measurement time. The source was placed at different positions within the active field of view (FOV). The spatial resolution, measured radially and tangentially, was obtained by applying the 2D filtered back-projection (FBP) within the software for tomographic image reconstruction (STIR) toolkit [48]. Spatial resolution varied between $2.34 \mathrm{~mm}$ and $3.66 \mathrm{~mm}$ (FWHM) axially moving radially $10 \mathrm{~mm}$ to $100 \mathrm{~mm}$ from the centre of the FOV.

\section{Sensitivity}

The simulated coincidence efficiency (i.e., the sensitivity) of the PET scanner for a point source positioned at the centre of the FOV was $65 \mathrm{cps} / \mathrm{kBq}$.

\section{Count rate}

In order to assess the count rates, we used a solid, cylindrical phantom made of polyethylene (density $0.96 \pm 0.1 \mathrm{~g} / \mathrm{cm}^{3}$ ) with dimensions of $70 \mathrm{~mm}$ in length and $25 \mathrm{~mm}$ in diameter, according to NEMA NU 4-2008. The phantom is placed at the centre of the axial and transaxial FOV of the modelled scanner. A cylindrical hole of $3.2 \mathrm{~mm}$ diameter was drilled parallel to the central axis of the cylinder, at a radial distance of $10 \mathrm{~mm}$ from the centre. The line source insert is a clear polyethylene plastic tube $60 \mathrm{~mm}$ in length, filled with 5 to $11 \mathrm{kBq} / \mathrm{ml}$ of ${ }^{18} \mathrm{~F}$ and threaded through the hole in the phantom for $1000 \mathrm{sec}$ measurement time. The noise equivalent count (NEC) curve derived from the NEMA NU2-2008 phantom shows a peak NEC above $1.8 \mathrm{Mcps}$ at $250 \mathrm{MBq}$.

\subsubsection{Attenuation correction}

Attenuation correction is essential for quantitative PET and is more challenging than in standard PET/CT. To optimize processing, we again used data obtained with existing PET/MR instrumentation (mMR in Munich). We developed a method that is based on the calculation of the R2-map [49] from the dual echo Ultra-Short-Time-Echo (dUTE) images, and further post-processing to extract the bone structures and air cavities. The rest of the tissue was assumed to be soft tissue (fat, water...). It has been demonstrated in previous studies that bone and air are the two main tissues to identify, that have an impact on the PET quantification [50]. The post-processing was based on statistical-based thresholding followed by linear transformation of the intensity values to Hounsfield units [49]. To evaluate the method, nine patients were double measured in a PET/CT scanner (for reference) and in the PET/MRI, and uptake concentration in several brain regions were compared. A further comparison of the results was performed with the two methods provided by the mMR manufacturer based on the Dixon and UTE sequences, and with three state-of-the-art methods [51-53], one of them being the method used in the pilot study at Jülich. The evaluation was performed at the level of PET uptake accuracy and at diagnostics imaging accuracy, by comparing the nine patients with a database of control subjects (Neurostats) to identify hypo-metabolic regions, indicators for dementia [36]. Results demonstrated $<5 \%$ error in PET quantification, and $>90 \%$ precision at identifying abnormal glucose metabolism in patients with suspected Alzheimer's disease, compared to attenuation correction based on CT. Translation of this method to the TRIMAGE scanner will be straightforward.

\section{Discussion and Conclusions}

Several data acquisition and processing methods have been developed while the TRIMAGE scanner development is still under way.

Results from the experiments in Munich with the FDOPA (Figure 7) demonstrate that regional analysis of the striatum can be feasibly used in a multi-modal platform. The evaluation shows that group differences between healthy controls and schizophrenic patients are well replicable. Due to the clear overlap between the two groups, a statement on the individual level is possible only to a limited extent. It is important to highlight the small size of these regions (i.e. accumbens as anatomical subregion and limbic as functional sub-region, which emphasizes the need for an excellent functionalanatomical co-registration, as provided by simultaneous PET/MRI acquisition. This feature is especially relevant in long PET scans, like the ones performed in the present study. No partial volume 
correction was performed. Additional investigations by correlating the PET and fMRI data are ongoing.

A primary, exploratory analysis of the data of the healthy and the schizophrenic patient was done in Jülich. The acquired datasets from the three modalities show alterations in the connectivity measures assessed via fMRI (Figure 8) and a change in binding potential in the PET data (Figure 9). The MMN LDAEP EEG data (Figure 10) show a difference between volunteer and schizophrenic patient data on a single trial level. We emphasize that in the absence of a group comparison, these data should not be over interpreted. Both the functional connectivity measures ReHo and DC showed less activation in comparison to the healthy subject. This indicates that communications between neurons in the brain of the schizophrenic patient are disrupted locally and at long range. Interestingly, the joint pilot data from Munich and Jülich show alterations in the nucleus accumbens. An ROI analysis performed on the Jülich data also displayed alterations in the glutamatergic system in the schizophrenic patient; we note this is a single subject result and should not be over interpreted. This avenue will be further investigated.

These preliminary results validate the PET $\left[{ }^{11} \mathrm{C}\right]-\mathrm{ABP} 688$ bolus + infusion scheme and the design of MMN paradigm. The primary results from the three modalities show significant difference in $\mathrm{BP}_{\mathrm{ND}}$ in the considered target regions (PET), difference in amplitude of MMN (EEG) and significant difference in functional connectivity measures in AN and SN (fMRI) between a healthy control subject and a schizophrenic patient indicating the potential use of a simultaneous trimodal measurement as a tool for detecting biomarkers in schizophrenia.

These findings need replication and will be extended to a much larger sample, as planned. However, initial findings on single subject level are very encouraging.

The TRIMAGE scanner development, the PET and MR scanner have been designed and built both as for the hardware and software side. The preliminary performance is very encouraging, since they respect the original specifications. The integration of three devices (PET, MR and EEG) in a single instrument is ongoing and the complete TRIMAGE scanner will be installed in Jülich in late spring 2018 to perform the pilot study on the 3 groups of 15 schizophrenic patients, 15 prodromic patients and 15 healthy volunteers

The major advantage of the magnet is the new superconducting technology developed with a cryocooler with no liquid nitrogen and a closed circuit He gas. This allows a much simpler and safer installation and an easier and cheaper maintenance. Furthermore, the limited axial extension reduces claustrophobia to the patient and allows bolus injection with direct supervision. The limited field strength of $1.5 \mathrm{~T}$ could give a lower signal/noise ratio with respect to a $3 \mathrm{~T}$ magnet, but this will be compensated by a more powerful dedicated RF coil. The PET scanner performs well beyond the state of the art. The spatial resolution of $2.34 \mathrm{~mm}$ (FWHM) axially as obtained at $10 \mathrm{~mm}$ radius from the centre of the FOV with 2D-FBP is almost a factor 2 better than the current mMR whole body PET/MR system [27]. The improvement in spatial resolution is of outmost importance for obtaining quantitative information within the sub-regions of the striatum (i.e., Figure 6 and Figure 9), because a higher spatial resolution increases the recovery coefficient. Furthermore, the simulated sensitivity of the PET scanner for a point-like source of $10 \mathrm{MBq}$ at the centre of the FOV is $6.1 \%$ for a $380-$ $850 \mathrm{keV}$ energy window, i.e., at least a factor 2 higher than the current PET/MR systems. On the other hand, the limited axial extend of $160 \mathrm{~mm}$ is lower than the standard $250 \mathrm{~mm}$ of the commercial PET/MR devices, but the available axial extent is adequate for the designed application. The addition of the EEG adds the third imaging modality to the TRIMAGE scanner. This is a unique feature that could give a complete temporal analysis. Once available, this new trimodality instrumentation may facilitate hypotheses for new biomarkers, which can be checked versus clinical evidence not only in the field of schizophrenia, but also in other mental disorders. 


\section{Vitae}

\subsection{Alberto Del Guerra}

He has been professor of Medical Physics at Napoli University, Ferrara University and Pisa University, Head of the Functional Imaging and Instrumentation Group and Director of the Specialty School of Medical Physics of Pisa University, visiting scientist at LBL and visiting Professor at University of Washington. He is now adjunct Professor of Medical Physics and coordinator of the TRIMAGE project.

\subsection{Salleh Ahmad}

CTO of Weeroc s.a.s. He worked for Omega microelectronics group (IN2P3/CNRS). His area of expertise is mixed signal ASIC design. Salleh holds a PhD in microlectronics from Université Paris Sud 11.

\subsection{Mihai Avram}

Postdoctoral researcher at the Medical Faculty of the Technical University Munich and Neuroimaging Center. $\mathrm{PhD}$ in human biology/neuroscience with focus on the imaging of human decisionmaking in 2004 at the Ludwig-Maximilian's-University, Munich.

\subsection{Nicola Belcari}

Associate Professor of Medical Physics at the University of Pisa. He received the Ph.D. in Applied Physics in 2003. His research activity is focused on the development of new imaging detectors for preclinical molecular imaging and brain PET/MR.

\subsection{Arne Berneking}

Electrical engineer graduated at RWTH Aachen University with major medical engineering. Currently, he works at Bruker BioSpin and he is finishing his $\mathrm{PhD}$ thesis on Novel Hardware for Hybrid MR/PET Imaging.

\subsection{Laura Biagi}

Ph.D. in Physics at the University of Pisa. She works on MR neuroimaging from 2002. Her main research is focused on the study of cerebral metabolism and function, as well the structural imaging at very high resolution, with particular interest in paediatric populations and disease. 


\subsection{Maria Giuseppina Bisogni}

Associate professor of Medical Physics at the University of Pisa. PhD in Physics in 1998 and specialist in Medical Physics in 1999. Her research activity is focused on the study of solid state detectors and their application to medical imaging, with a special focus on PET in hadrontherapy.

\subsection{Felix Brandl}

He studied Medicine at Ludwig-Maximilians-Universität in Munich (Germany), resulting in an MD degree, and is now pursuing a $\mathrm{PhD}$ in Neuroscience at Technische Universität München.

\subsection{Jorge Cabello}

Graduated in Electronics Engineering at University of Alcala de Henares (Spain). Later he obtained his Ph.D. on medical imaging at University of Surrey (United Kingdom). He is currently researcher in Nuclear Medicine at Klinikum rechts der Isar (Germany). His research interests are image reconstruction and kinetic analysis in PET/MRI.

\subsection{Niccolò Camarlinghi}

$\mathrm{PhD}$ in applied physics at Pisa University in 2012. He is currently working as researcher at Pisa University in the field of Positron Emission Tomography (PET).

\subsection{Piergiorgio Cerello}

$\mathrm{Ph} . \mathrm{D}$. in experimental physics in 1995, he was among the founders of the ALICE project at the CERN LHC. In the past 15 years, he was involved in several medical physics projects focused on the development of algorithms for computer assisted detection and of innovative PET detectors.

\subsection{Chang-Hoon Choi}

He obtained his PhD in MRI Physics at the University of Aberdeen, UK in 2010 and worked for MR Solutions, UK until 2014. He is an MRI expert and has been working in Forschungszentrum Jülich, Germany as a research scientist since 2014.
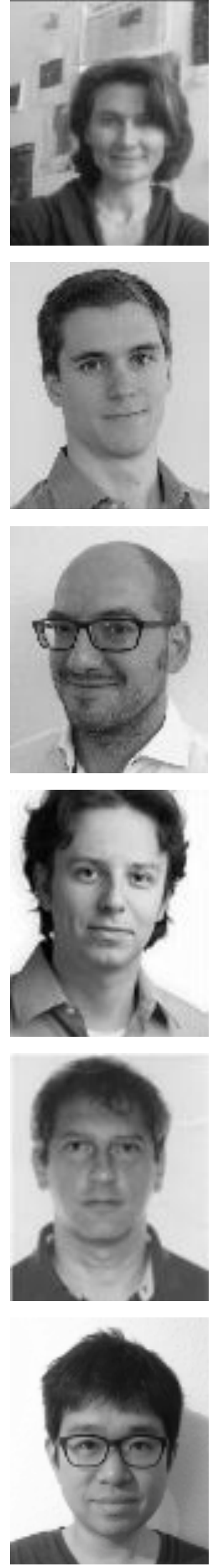


\subsection{Silvia Coli}

Graduated in Aerospace Engineering at Politecnico of Turin. Technologist at INFN (TURIN section) since 2000. Responsible for Cooling System project for ALICE-ITS, PANDA-MVD and TRIMAGE projects.

\subsection{Sabrina Colpo}

Graduated in Electronic Engineering at University Federico II, Naples (Italy), in 2005. From 2006 to 2012 she joined the RF-MEMS group at FBK, Trento (Italy). Currently, she is responsible for the production at AdvanSiD srl, focused on advanced silicon radiation detectors technology.

\subsection{Julien Fleury}

CEO and founder of Weeroc s.a.s. He has been working for a RF company in Santa Clara US and then with Omega microelectronics group (IN2P3/CNRS). He has expertise in analog and mixed signal microelectronics design and holds an engineering degree.

\subsection{Vito Gagliardi}

Master degree in Physics in 2015 with a thesis on PET/MRI magnetic compatibility, he is attending the Specialization School of Medical Physics at the University of Pisa. His main research is on MR imaging and RF coil design.

\subsection{Giuseppe Giraudo}

Senior technologist at INFN Torino. Supervisor of the mechanical structure and system integration of ITS Alice at CERN LHC, MVD Panda at GSI-FAIR and INSIDE In Beam PET at CNAO. Advisor/member of the Technical Board of Panda Project at GSI-FAIR. Cooling system design for FEE CGEM for BESIII at IHEP.

\subsection{Karsten Heekeren}

Karsten Heekeren studied medicine at RWTH Aachen University. After his specialist training he joined the University Hospital of Psychiatry Zurich as a senior physician. Currently, he is head of the Department of Psychiatry and Psychotherapy at St. Josef Hospital in Moers and adjunct professor at the University of Zurich. 


\subsection{Wolfram Kawohl}

Medical doctor degree at the University of Aachen. Board-certified psychiatrist and psychotherapist since 2005 . He is an adjunct professor of psychiatry and psychotherapy with the University of Zurich and head of the Department of Psychiatry and Psychotherapy at the Psychiatric Services of Aargovia, University of Zurich.

\subsection{Theodora Kostou}

Graduated from the Physics Department, University of Patras in 2012. In 2014, she received her Master's degree in Medical Physics at the Department of Medical Physics, School of Medicine, University of Patras, where she is currently doing her $\mathrm{PhD}$ studies.

\subsection{Jean-Luc Lefaucheur}

Graduated at the Ecole Centrale and Strathclyde University, he has worked many years in the development of detectors for PET such as LSO and LYSO as well as in the field of imaging systems. He is CEO of the company Inviscan SAS who develops, manufactures and markets imaging systems for research in medical applications.

\subsection{Christoph Lerche}

Ph.D. in Experimental Atomic, Molecular and Particle Physics at the University of Valencia, Spain in 2006. Head of the PET Physics Group at the Institute of Neuroscience and Medicine-4 (Medical Imaging Physics) of the Forschungszentrum Jülich GmbH, Germany since 2014. Concurrent PET/MR and PET/MR/EEG imaging is the primary focus of his research.

\subsection{George Loudos}

Assistant Professor at Department of Biomedical Engineering at TEI Athens. He has participated as a coordinator or partner in more than 15 EU projects. His research is on medical instrumentation, molecular imaging of nanoparticles and simulations for optimization of diagnostic and therapeutic protocols.

\subsection{Matteo Morrocchi}

$\mathrm{PhD}$ in 2015 at the University of Pisa working on high spatial resolution PET detectors using SiPMs coupled to monolithic or pixelated scintillators. He is currently a post-doctoral researcher working on data acquisition systems for PET and on applications of SiPM detectors.
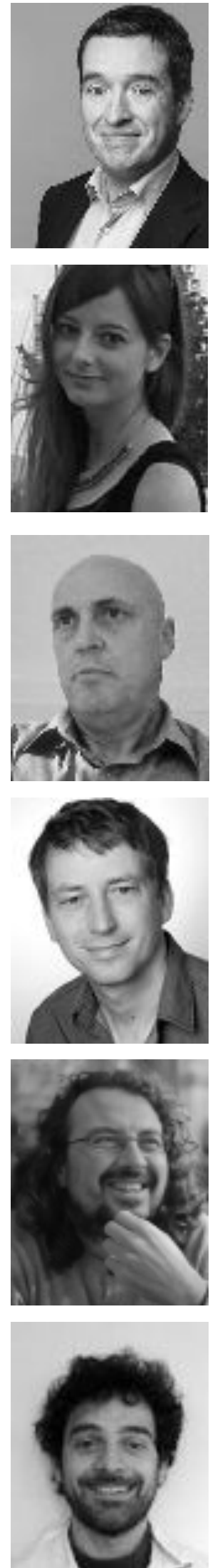


\subsection{Julien Muller}

Chief Technology Officer at RS2D, manager of R\&D project. His background is electronic engineering and he has 12 years of experience in developing electronic and software for NMR and MRI and 6 years of experience with cryogen free superconducting magnet.

\subsection{Mona Mustafa}

MD, Senior Clinical Physician, Dpt. of Nuclear Medicine at TUM. Expert in PET in dementia, neuro-oncology and oncology. She has 10 years of experience with high-throughput PET centres.

\subsection{Irene Neuner}

Board certified physician in psychiatry and neurology. She holds a double affiliation as deputy head of the Department of Psychiatry, Psychotherapy and Psychosomatics, RWTH Aachen University, Germany and as Associate Professor for Multimodal Imaging at the RWTH Aachen and the Forschungszentrum Jülich GmbH within the framework of JARA BRAIN (Juelich-Aachen-Research Alliance).

\subsection{Panagiotis Papadimitroulas}

Medical physicist, graduated from the School of Applied Mathematical and Physical Sciences, National Technical University of Athens. His research interests lie in the field of Monte Carlo simulations on personalized medicine for imaging and dosimetry applications. He has participated in 12 national and international projects including FP7, H2020, COST.

\subsection{Francesco Pennazio}

$\mathrm{PhD}$ in physics in 2013 at the University of Torino. His research activity at INFN Torino focuses on Monte Carlo simulations and experimental data analysis of multi-modality PET and in-beam PET systems.

\subsection{Ravichandran Rajkumar}

Bachelor degree in Biomedical Engineering. PhD student at the Jülich Research Center and University Hospital Aachen, Germany. His research interests include multimodal neuro-imaging and image based biomarkers for brain disorders.
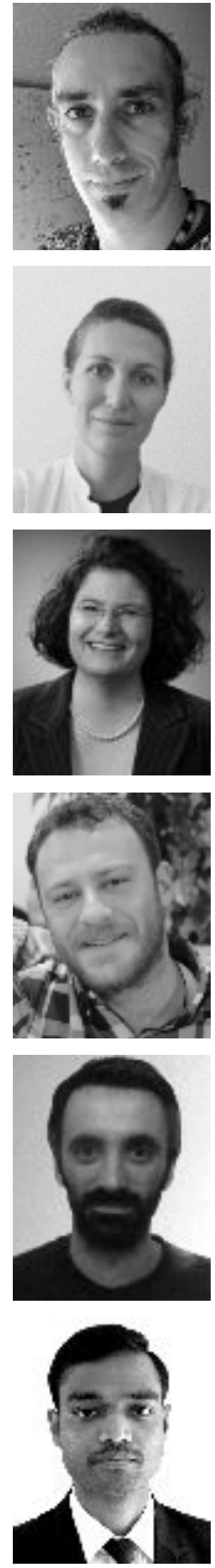


\subsection{Cláudia Régio Brambilla}

Medical Physicist and Master in Health Sciences. PhD student at Institute of Neuroscience and Medicine, INM-4, Forschungszentrum Jülich GmbH.

\subsection{Julien Rivoire}

Graduated from Lyon University in Biomedical engineering. Scientist at the university of Mainz and University of California San Francisco on Hyperpolarized Helium3 MRI and cartilage imaging. He is now MRI application manager at RS2D.

\subsection{Elena Rota Kops}

Senior physicist in the PET group of the Institute of Neuroscience and Medicine (INM) at the Forschungszentrum Jülich. She studied physics at the University of Turin and graduated in Plasma Physics at the University of Düsseldorf. Since her PhD she is staff member of the PET group.

\subsection{Jürgen Scheins}

$\mathrm{PhD}$ in Experimental Particle Physics at DESY Hamburg, Germany; since 2004 in the PET working group of INM at Forschungszentrum Jülich, since 2008 staff member.

\subsection{Rémy Schimpf}

Master degree in chemistry. He worked at Bruker Biospin France, as application Engineer, NMR product manager and finally as sales and marketing Director. He is president and founder of RS2D since 2003. RS2D is a leading company worldwide in providing OEM solutions for MRI and NMR applications.

\subsection{Jon Shah}

Following his $\mathrm{PhD}$ at the University of Manchester, Jon Shah went to Japan where he worked on the development of methods for MRI and spectroscopy. He worked at the University of Cambridge on MR and then in Germany where he now works at the FZ Jülich. Currently he is Director of the INM-4 and INM-11 in Jülich, Professor of MRI Physics in Aachen and a Distinguished Professor at the MIME in Melbourne.
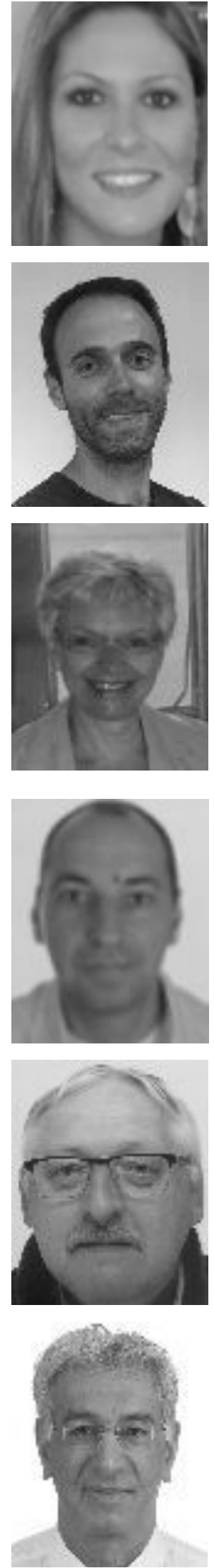


\subsection{Christian Sorg}

He studied human medicine and mathematics in Berlin and Munich. Trained as a psychiatrist at Department of Psychiatry of Technische Universtät München TUM, Germany. Currently he is head of the 'Neuropsychiatry and Neuroimaging Lab' at TUM Neuroimaging Center of Departments of Psychiatry and Neuroradiology.

\subsection{Giancarlo Sportelli}

$\mathrm{Ph} . \mathrm{D}$. degree from the Telecommunications Engineering School of the Universidad Politécnica de Madrid in 2010. He is now researcher of the Department of Physics, University of Pisa. His expertise is in digital electronics, semiconductor technology and their application to PET and $\mathrm{PET} / \mathrm{MR}$.

\subsection{Michela Tosetti}

Coordinator of the technical research activities of the 7T MR system installed at the IMAGO7 Foundation in Pisa. Her expertise is focused on advanced Magnetic Resonance techniques, and their applications on humans, with the specific interest is to understand cerebral and muscular mechanisms at various levels (structure, metabolism, function and connectivity).

\subsection{Riccardo Trinchero}

$\mathrm{Ph} . \mathrm{D}$. degree in electronics and communication engineering from Politecnico di Torino, Torino, Italy, in 2015. He is currently an Assistant Professor with the Politecnico di Torino. His research interests include the analysis of time-varying systems, modelling and simulation of switching converters, statistical simulation and EMC design.

\subsection{Christine Wyss}

$\mathrm{PhD}$ at the University Hospital of Psychiatry Zürich on the methodology of electrophysiological biomarkers in Psychiatry. She is investigating the physiological and chemical mechanisms during the processing of auditory stimulation for the TRIMAGE project.

\subsection{Sibylle Ziegler}

$\mathrm{PhD}$ in 1989 at the German Cancer Center in Heidelberg. She carried out her research on multimodal imaging at the Technical University Munich, and since 2016 at the Department of Nuclear Medicine of the University Hospital at Ludwig-Maximilians-University in Munich. She is member of the review board of the German Research Foundation DFG and of the National Academy of Science and Engineering.
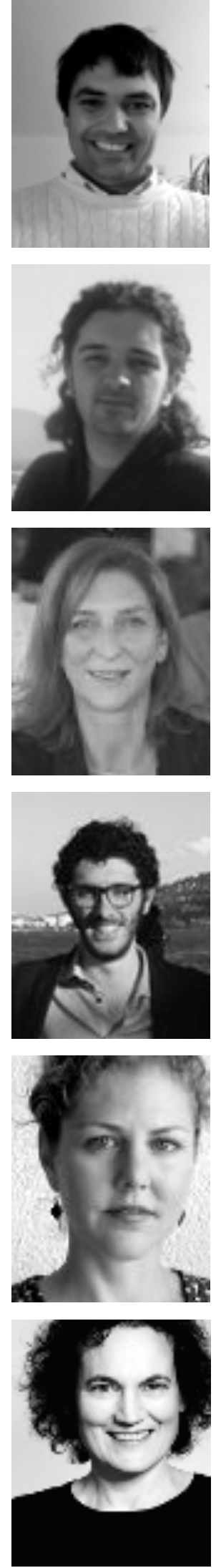


\section{Acknowledgments}

The research leading to these results has received funding from the European Union Seventh Framework Programme (FP7/2007-2013) under grant agreement $n^{\circ} 602621$ - Trimage.

$\mathrm{N}$. Jon Shah is funded in part by the Helmholtz Alliance ICEMED - Imaging and Curing Environmental Metabolic Diseases, through the Initiative and Network Fund of the Helmholtz Association. N. Jon Shah acknowledges funding from the BMBF and Siemens for the 9.4T MR-PET project (Grant number 13N9121). N. Jon Shah and Irene Neuner report partial funding from the DFG for the implementation of the trimodal approach (Shah DFG SH 79/2-2). Claudia Regio-Brambilla is financed by a DAAD PhD stipend under the joint supervision of Irene Neuner and Christoph Lerche with focus on the ABP688 approach. We thank Suzanne Schaden, Silke Frensch and Cornelia Frey for excellent technical assistance in data acquisition in Jülich.

The PET/MR facility at the Technische Universität München was funded by the Deutsche Forschungsgemeinschaft, Großgeräteinitiative (DFG). The research leading to these results has received funding from the European Union Seventh Framework Program (FP7) under grant agreement no. 294582 ERC Grant MUMI. The excellent technical assistance of Sylvia Schachoff at TUM is greatly appreciated. 


\section{7- REFERENCES}

[1] Liddle PF, Lane CJ, Ngan ET. Immediate effects of risperidone on cortico-striato - thalamic loops and the hippocampus. Br J Psychiatry 2000;177:402-407.

[2] Nuechterlein KH, Subotnik KL, Ventura J, Green MF, Gretchen-Doorly D, Asarnow RF. The puzzle of schizophrenia: tracking the core role of cognitive deficits. Dev Psychopathol 2012;24:529-536.

[3] Barch DM, Ceaser A. Cognition in schizophrenia: core psychological and neural mechanisms. Trends Cogn Sci 2012;16:27-34.

[4] Penn DL, Sanna LJ, Roberts DL. Social cognition in schizophrenia: an overview. Schizophr Bull 2008;34:408-411.

[5] Howes OD, Williams M, Ibrahim K, Leung G, Egerton A, McGuire PK, et al. Midbrain dopamine function in schizophrenia and depression: a post-mortem and positron emission tomographic imaging study. Brain 2013;136:3242-51. doi:10.1093/brain/awt264.

[6] Weinberger DR. Implications of normal brain development for the pathogenesis of schizophrenia. Arch Gen Psychiatry 1987;44:660-669.

[7] Davis KL, Kahn RS, others. Dopamine in schizophrenia: a review and reconceptualization. Am J Psychiatry 1991;148:1474.

[8] Carlsson A. The current status of the dopamine hypothesis of schizophrenia. Neuropsychopharmacology 1988.

[9] Wyss C, Hitz K, Hengartner MP, Theodoridou A, Obermann C, Uhl I, et al. The loudness dependence of auditory evoked potentials (LDAEP) as an indicator of serotonergic dysfunction in patients with predominant schizophrenic negative symptoms. PLoS One 2013;8:e68650.

[10] Gouzoulis-Mayfrank E, Heekeren K, Neukirch A, Stoll M, Stock C, Obradovic M, et al. Psychological effects of (S)-ketamine and N, N-dimethyltryptamine (DMT): a double-blind, cross-over study in healthy volunteers. Pharmacopsychiatry 2005;38:301-311.

[11] Singh SP, Singh V. Meta-analysis of the efficacy of adjunctive NMDA receptor modulators in chronic schizophrenia. CNS Drugs 2011;25:859-885.

[12] Tuominen HJ, Tiihonen J, Wahlbeck K. Glutamatergic drugs for schizophrenia. Cochrane Database Syst Rev 2006;2.

[13] Lodge DJ, Grace AA. Hippocampal dysregulation of dopamine system function and the pathophysiology of schizophrenia. Trends Pharmacol Sci 2011;32:507-513.

[14] Sullivan EM, O’Donnell P. Inhibitory interneurons, oxidative stress, and schizophrenia. Schizophr Bull 2012;38:373-376.

[15] Rotaru DC, Lewis DA, Gonzalez-Burgos G. The role of glutamatergic inputs onto parvalbuminpositive interneurons: relevance for schizophrenia. Rev Neurosci 2012;23:97-109.

[16] Strasser HC, Lilyestrom J, Ashby ER, Honeycutt NA, Schretlen DJ, Pulver AE, et al. Hippocampal and ventricular volumes in psychotic and nonpsychotic bipolar patients compared with schizophrenia patients and community control subjects: a pilot study. Biol Psychiatry 2005;57:633-639.

[17] Del Guerra A, Belcari N, Bisogni M. Positron Emission Tomography: Its 65 years. Nuovo Cimento Riv Ser 2016;39:155-223. doi:10.1393/ncr/i2016-10122-6.

[18] Takahashi H, Rissling AJ, Pascual-Marqui R, Kirihara K, Pela M, Sprock J, et al. Neural substrates of normal and impaired preattentive sensory discrimination in large cohorts of nonpsychiatric subjects and schizophrenia patients as indexed by MMN and P3a change detection responses. Neuroimage 2013;66:594-603.

[19] Ehlis A-C, Pauli P, Herrmann MJ, Plichta MM, Zielasek J, Pfuhlmann B, et al. Hypofrontality in schizophrenic patients and its relevance for the choice of antipsychotic medication: an eventrelated potential study. World J Biol Psychiatry 2012;13:188-199. 
[20] Kawohl W, Waberski TD, Darvas F, Norra C, Gobbelé R, Buchner H. Comparative source localization of electrically and pressure-stimulated multichannel somatosensory evoked potentials. J Clin Neurophysiol 2007;24:257-262.

[21] Heekeren K, Daumann J, Neukirch A, Stock C, Kawohl W, Norra C, et al. Mismatch negativity generation in the human 5HT2A agonist and NMDA antagonist model of psychosis. Psychopharmacology (Berl) 2008;199:77-88.

[22] Kenemans JL, Kähkönen S. How human electrophysiology informs psychopharmacology: from bottom-up driven processing to top-down control. Neuropsychopharmacology 2011;36:26.

[23] Shah NJ, Oros-Peusquens A-M, Arrubla J, Zhang K, Warbrick T, Mauler J, et al. Advances in multimodal neuroimaging: hybrid MR-PET and MR-PET-EEG at 3T and 9.4 T. J Magn Reson 2013;229:101-15. doi:10.1016/j.jmr.2012.11.027.

[24] Rajkumar R, Rota Kops E, Mauler J, Tellmann L, Lerche C, Herzog H, et al. Simultaneous trimodal PET-MR-EEG imaging: Do EEG caps generate artefacts in PET images? PLOS ONE 2017;12:e0184743. doi:10.1371/journal.pone.0184743.

[25] Del Guerra A. TRIMAGE - Development of a Simultaneous Trimodal (PET/MR/EEG) Imaging Tool for Early Diagnosis of Schizophrenia and Other Mental Disorders. Eur Psychiatry 2015;30:161. doi:10.1016/S0924-9338(15)30132-2.

[26] Zaidi H, Del Guerra A. An outlook on future design of hybrid PET/MRI systems. Med Phys 2011;38:5667-89. doi:10.1118/1.3633909.

[27] Delso G, Fürst S, Jakoby B, Ladebeck R, Ganter C, Nekolla SG, et al. Performance Measurements of the Siemens mMR Integrated Whole-Body PET/MR Scanner. J Nucl Med 2011;52:1914-22. doi:10.2967/jnumed.111.092726.

[28] Lammertsma AA, Hume SP. Simplified reference tissue model for PET receptor studies. Neuroimage 1996;4:153-158.

[29] Kumakura Y, Vernaleken I, Gründer G, Bartenstein P, Gjedde A, Cumming P. PET Studies of Net Blood-Brain Clearance of FDOPA to Human Brain: Age-Dependent Decline of [18F]Fluorodopamine Storage Capacity. J Cereb Blood Flow Metab 2005;25:807-19. doi:10.1038/sj.jcbfm.9600079.

[30] Hintermann S, Vranesic I, Allgeier H, Brülisauer A, Hoyer D, Lemaire M, et al. ABP688, a novel selective and high affinity ligand for the labeling of mGlu5 receptors: identification, in vitro pharmacology, pharmacokinetic and biodistribution studies. Bioorg Med Chem 2007;15:903-914.

[31] Neuner I, Kaffanke JB, Langen K-J, Rota Kops E, Tellmann L, Stoffels G, et al. Multimodal imaging utilising integrated MR-PET for human brain tumour assessment. Eur Radiol 2012;22:2568-2580.

[32] Shah NJ, Arrubla J, Rajkumar R, Farrher E, Mauler J, Rota Kops E, et al. Multimodal Fingerprints of Resting State Networks as assessed by Simultaneous Trimodal MR-PET-EEG Imaging. Sci Rep 2017;7.

[33] Carson RE, Channing M a, Blasberg RG, Dunn BB, Cohen RM, Rice KC, et al. Comparison of bolus and infusion methods for receptor quantitation: application to [18F]cyclofoxy and positron emission tomography. J Cereb Blood Flow Metab 1993;13:24-42. doi:10.1038/jcbfm.1993.6.

[34] Burger C, Deschwanden A, Ametamey S, Johayem A, Mancosu B, Wyss M, et al. Evaluation of a bolus/infusion protocol for 11C-ABP688, a PET tracer for mGluR5. Nucl Med Biol 2010;37:845-51. doi:10.1016/j.nucmedbio.2010.04.107.

[35] Rota Kops E, Hautzel H, Herzog H, Antoch G, Shah NJ. Comparison of template-based versus CT-based attenuation correction for hybrid MR/PET scanners. IEEE Trans Nucl Sci 2015;62:2115-2121.

[36] Cabello J, Lukas M, Rota Kops E, Ribeiro A, Shah NJ, Yakushev I, et al. Comparison between MRI-based attenuation correction methods for brain PET in dementia patients. Eur J Nucl Med Mol Imaging 2016;43:2190-2200. 
[37] Del Guerra A, Belcari N, Giuseppina Bisogni M, Corsi F, Foresta M, Guerra P, et al. Silicon Photomultipliers (SiPM) as novel photodetectors for PET. Nucl Instrum Methods Phys Res Sect Accel Spectrometers Detect Assoc Equip 2011;648, Supplement 1:S232-5. doi:10.1016/j.nima.2010.11.128.

[38] Camarlinghi N, Belcari N, Cerello P, Pennazio F, Sportelli G, Zaccaro E, et al. Evaluation of Algorithms for Photon Depth of Interaction Estimation for the TRIMAGE PET Component. IEEE Trans Nucl Sci 2016;63:70-4. doi:10.1109/TNS.2015.2512986.

[39] Ahmad S, Fleury J, Taille C de la, Seguin-Moreau N, Dulucq F, Martin-Chassard G, et al. Triroc: A Multi-Channel SiPM Read-Out ASIC for PET/PET-ToF Application. IEEE Trans Nucl Sci 2015;62:664-8. doi:10.1109/TNS.2015.2397973.

[40] Sportelli G, Ahmad S, Belcari N, Bisogni MG, Camarlinghi N, Pasquale AD, et al. The TRIMAGE PET Data Acquisition System: Initial Results. IEEE Trans Radiat Plasma Med Sci 2017;1:168-77. doi:10.1109/TNS.2016.2633237.

[41] Berneking A, Trinchero R, Ha Y, Finster F, Cerello P, Lerche C, et al. Design and Characterization of a Gradient-Transparent RF Copper Shield for PET Detector Modules in Hybrid MR-PET Imaging. IEEE Trans Nucl Sci 2017;64:1118-1127.

[42] Jan S, Benoit D, Becheva E, Carlier T, Cassol F, Descourt P, et al. GATE V6: a major enhancement of the GATE simulation platform enabling modelling of CT and radiotherapy. Phys Med Biol 2011;56:881.

[43] Scheins JJ, Herzog H, Shah NJ. Fully-3D PET Image Reconstruction Using ScannerIndependent, Adaptive Projection Data and Highly Rotation-Symmetric Voxel Assemblies. IEEE Trans Med Imaging 2011;30:879-92. doi:10.1109/TMI.2011.2109732.

[44] Herzog H, Langen K-J, Weirich C, Rota Kops E, Kaffanke J, Tellmann L, et al. High resolution BrainPET combined with simultaneous MRI. Nuklearmedizin 2011;50:74-82. doi:10.3413/Nukmed-0347-10-09.

[45] National Electrical Manufacturers Association. Standards Publication NU-2-1994: performance measurements of positron emission tomography. Wash DC Natl Electr Manuf Assoc 1994.

[46] National Electrical Manufacturers Association. Standards Publication NU-2-2001: performance measurements of positron emission tomography. Rosslyn VA Natl Electr Manuf Assoc 2001.

[47] National Electrical Manufacturers Association. Standards Publication NU-4-2008: performance measurements of small animal positron emission tomographs. Wash DC Natl Electr Manuf Assoc 2008.

[48] Thielemans K, Tsoumpas C, Mustafovic S, Beisel T, Aguiar P, Nikolaos Dikaios, et al. STIR: software for tomographic image reconstruction release 2. Phys Med Biol 2012;57:867. doi:10.1088/0031-9155/57/4/867.

[49] Cabello J, Lukas M, Förster S, Pyka T, Nekolla SG, Ziegler SI. MR-based attenuation correction using ultrashort-echo-time pulse sequences in dementia patients. J Nucl Med 2015;56:423-429.

[50] Visvikis D, Monnier F, Bert J, Hatt M, Fayad H. PET/MR attenuation correction: where have we come from and where are we going? Eur J Nucl Med Mol Imaging 2014;41:1172-5. doi:10.1007/s00259-014-2748-0.

[51] Burgos N, Cardoso MJ, Thielemans K, Modat M, Pedemonte S, Dickson J, et al. Attenuation Correction Synthesis for Hybrid PET-MR Scanners: Application to Brain Studies. IEEE Trans Med Imaging 2014;33:2332-41. doi:10.1109/TMI.2014.2340135.

[52] Izquierdo-Garcia D, Hansen AE, Förster S, Benoit D, Schachoff S, Fürst S, et al. An SPM8based approach for attenuation correction combining segmentation and nonrigid template formation: application to simultaneous PET/MR brain imaging. J Nucl Med 2014;55:18251830.

[53] Santos Ribeiro A, Rota Kops E, Herzog H, Almeida P. Hybrid approach for attenuation correction in PET/MR scanners. Nucl Instrum Methods Phys Res Sect Accel Spectrometers Detect Assoc Equip 2014;734:166-170. 


\section{Supplemental Files}

\subsection{Analysis of the Jülich data}

\section{fMRI data Analysis}

fMRI data analysis was performed using a Matlab-based toolkit, DPABI [1]. DPABI contains libraries for processing fMRI data that depend on another Matlab-based software package, Statistical Parametric Mapping 12 (SPM12, Wellcome Trust Centre for Neuroimaging). The short range functional connectivity measure called regional homogeneity (ReHo) [2] and long range functional connectivity measure called degree centrality (DC) [3] were computed in fMRI data acquired during MMN task. Functional connectivity measures were computed after pre-processing steps which includes corrections for slice timing, motion and nuisance signal. A temporal filtering was performed between 0.01 to $0.08 \mathrm{~Hz}$. ReHo (voxel cluster size 27) and DC values (correlation cutoff - Pearson $\mathrm{r}$ $>0.25, \mathrm{p}<0.0001)$ were calculated in subject's native space and were linearly standardized to $\mathrm{Z}$ values. $Z$ standardized connectivity measures were then normalized to standard MNI space $\left(2 \mathrm{~mm}^{3}\right)$. In-order to quantify the changes in connectivity values between the healthy and schizophrenic subjects, voxel values were extracted from whole brain grey matter (GM) mask and functional masks such as the auditory and salience networks for comparison. Whole brain GM mask was made by segmenting MNI152 brain template using FSL FEAT [4] software package. Finally, GM mask was created from segmented GM image voxels which have a higher than $50 \%$ probability. Functional masks were obtained from 90 fROI atlas [5]. The functional masks (auditory network (AN) and salience network (SN) masks) were corrected for GM using the GM mask created this was done in order to consider only the voxels in the GM region for further analyses.

A two-sample t-test was performed in Matlab (Version 8.5, Matworks) to compare functional connectivity measures between GM region and AN and SN within subjects. Similarly, a two-sample t-test was performed compare functional connectivity measures in GM, AN and SN between healthy subject and schizophrenic patient.

\section{PET data Analysis}

PET images were smoothed with an isotropic Gaussian filter $(3 \mathrm{~mm})$, motion corrected and registered to MRI, the activity concentration was extracted from the precuneus, cingulum posterior part, hippocampus and parahippocampus, nucleus accumbens (volume-of-interest sphere of $8 \mathrm{~mm}$ radius), middle and inferior frontal and cerebellum cortices (PMOD software package, version 3.5).

The binding potential was calculated during MMN task period (at equilibrium). The non-displaceable binding potential was calculated as $\mathrm{BP}_{\mathrm{ND}}=\left(\mathrm{C}_{\mathrm{t}}-\mathrm{C}_{\text {cer }}\right) / \mathrm{C}_{\mathrm{cer}}$, where $\mathrm{C}_{\mathrm{t}}$ represents the activity concentration in the brain tissue regions and $\mathrm{C}_{\text {cer }}$ is the reference tissue, in this case cerebellum.

A two-sample t-test between $\mathrm{BP}_{\mathrm{ND}}$ values of healthy volunteer and schizophrenic subject in different target regions was done using Matlab software package (version 8.5).

\section{EEG data Analysis}

EEG data were processed using EEGlab [6], a Matlab-based toolbox. The pre-processing steps included gradient artefact (GA) correction [7], down-sampling to $250 \mathrm{~Hz}$, additional filtering using a Butterworth zero-phase filter with lower cut-off frequency of $1 \mathrm{~Hz}$ and higher cut-off frequency of 8 $\mathrm{Hz}$ for the ECG channel and $20 \mathrm{~Hz}$ for other the EEG channels and ballistocardiogram (BCG) artefact correction [7]. An ICA-based decomposition was performed using the Infomax extended algorithm [8] to all 63 EEG channels. Further, artefact components were identified and removed using MARA toolbox [9]. EEG signals were re-referenced to an average reference and segmented between -400 
and $+600 \mathrm{~ms}$ based on standard and frequent auditory stimuli marker position. The segmented EEG data were averaged over trials. The amplitude between the $\mathrm{N} 1$ and the $\mathrm{P} 2$ peaks was calculated for standard tone and amplitude of $\mathrm{MMN}$ was calculated for deviant tone from the averaged trials in $\mathrm{Cz}$ channel.

\subsection{Recruitment and inclusion criteria}

Recruitment of subjects with schizophrenia diagnosis according to ICD-10 and age- and educationmatched healthy controls is described in the supplemental files. The recruitment took place at the two sites Jülich (between 16.03.2017-31.06.2017) and Munich (22.02.2017-31.07.2017), each one with a planned number of 20 patients and 20 controls. Inclusion criteria were identical for both study sites and are listed in the table below.

Inclusion criteria for all participants:

- age between 18 and 55 years

- no persons unable to give informed consent will be involved

- no mental retardation (IQ < 80) estimated with the WST (Wortschatztest) or MWT-B (tests for the premorbid intellectual performance level)

- no drug dependency (except of nicotine)

- no depressive or maniac episode, no bipolar disorder, no schizoaffective disorder, no PTSD

- no neurologic or other severe somatic disorder

- no contraindication for MRI or PET

Additional inclusion criteria for each group:

- for healthy controls:

- no current (or history of) mental disorder, checked with the operationalized Mini International Neuropsychiatric Interview (MINI)

- for subjects with manifest schizophrenia:

- diagnostic criteria according to ICD-10 are met.

- Patients in acute states of psychosis are excluded according to PANSS ratings $\leq 3$ in the subscales 'Delusions (P1)', 'Conceptual disorganisation (P2)', 'Hallucinatory behaviour (P3)', 'Mannerisms and posturing (G5)' and 'Unusual thought content (G9)'. (According to van Os et al., 2006[10])

- stable antipsychotic medication for at least 15 days 


\subsection{References for the supplemental files}

[1] Yan C-G, Wang X-D, Zuo X-N, Zang Y-F. DPABI: data processing \& analysis for (resting-state) brain imaging. Neuroinformatics 2016;14:339-351.

[2] Zang Y, Jiang T, Lu Y, He Y, Tian L. Regional homogeneity approach to fMRI data analysis 2004;22:394-400. doi:10.1016/j.neuroimage.2003.12.030.

[3] Buckner RL, Sepulcre J, Talukdar T, Krienen FM, Liu H, Hedden T, et al. Cortical hubs revealed by intrinsic functional connectivity: mapping, assessment of stability, and relation to Alzheimer's disease. J Neurosci 2009;29:1860-73. doi:10.1523/JNEUROSCI.5062-08.2009.

[4] Smith SM, Jenkinson M, Woolrich MW, Beckmann CF, Behrens TEJ, Johansen-Berg H, et al. Advances in functional and structural MR image analysis and implementation as FSL. NeuroImage 2004;23:208-19. doi:10.1016/j.neuroimage.2004.07.051.

[5] Shirer WR, Ryali S, Rykhlevskaia E, Menon V, Greicius MD. Decoding subject-driven cognitive states with whole-brain connectivity patterns. Cereb Cortex 2012;22:158-65. doi:10.1093/cercor/bhr099.

[6] Delorme A, Makeig S. EEGLAB: An open source toolbox for analysis of single-trial EEG dynamics including independent component analysis. J Neurosci Methods 2004;134:9-21. doi:10.1016/j.jneumeth.2003.10.009.

[7] Niazy RK, Beckmann CF, Iannetti GD, Brady JM, Smith SM. Removal of FMRI environment artifacts from EEG data using optimal basis sets. NeuroImage 2005;28:720-37. doi:10.1016/j.neuroimage.2005.06.067.

[8] Bell J, Sejnowski T. Information-Maximization Approach to Blind Separation and Blind Deconvolution. Technology 1995;1159:1129-59.

[9] Winkler I, Haufe S, Tangermann M. Automatic Classification of Artifactual ICA- Components for Artifact Removal in EEG Signals 2011:1-15.

[10] van Os J, Kenis G, Rutten BPF. The environment and schizophrenia. Nature 2010;468:203-12. doi:10.1038/nature09563. 